\title{
Dirac and Majorana neutrino signatures of Primordial Black Holes
}

\author{
Yuber F. Perez-Gonzalez
}

In collaboration with Cecilia Lunardini arXiv: 1910.XXXXX

Neutrino Platform Week 2019: Hot Topics in Neutrino Physics

CERN, October 11th, 2019

\section{\# Fermilab}

This manuscript has been authored by Fermi Research Alliance, LLC under Contract No. DEAC02-07CH11359 with the U.S. Department of Energy, Office of Science, Office of High

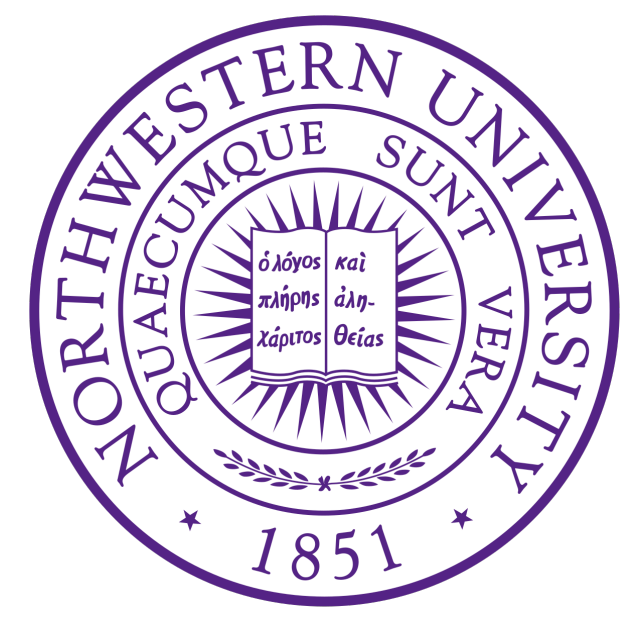




\section{Are there other mechanisms for neutrino emission?}




\section{Primordial Black Holes (PBH)}




\title{
Primordial Black Holes (PBH)
}

\author{
Astrophysical Black Holes \\ $M \gtrsim 1.4 M_{\odot}$
}




\section{Primordial Black Holes $(\mathrm{PBH})$}

Astrophysical Black Holes

$$
M \gtrsim 1.4 M_{\odot}
$$

\section{"Smaller" Black Holes}

\section{Larger densities}

$$
r_{S}=2 G M \quad M_{i} \sim \frac{t}{G} \sim 10^{15}\left(\frac{t}{10^{-23} \mathrm{~s}}\right) \mathrm{g}
$$




\section{Primordial Black Holes $(\mathrm{PBH})$}

Astrophysical Black Holes

$$
M \gtrsim 1.4 M_{\odot}
$$

"Smaller" Black Holes

\section{Larger densities}

$$
r_{S}=2 G M \quad M_{i} \sim \frac{t}{G} \sim 10^{15}\left(\frac{t}{10^{-23} \mathrm{~s}}\right) \mathrm{g}
$$

\section{Formation}

* Bubble collisions

* Pressure reduction

- Collapse of density fluctuations

Quantum effects

are important

$$
r_{S} \sim \lambda_{C}
$$

Black Holes evaporate by thermal emission

Hawking, 1975

Carr et al, 0912.5297 


\section{Primordial Black Holes $(\mathrm{PBH})$}

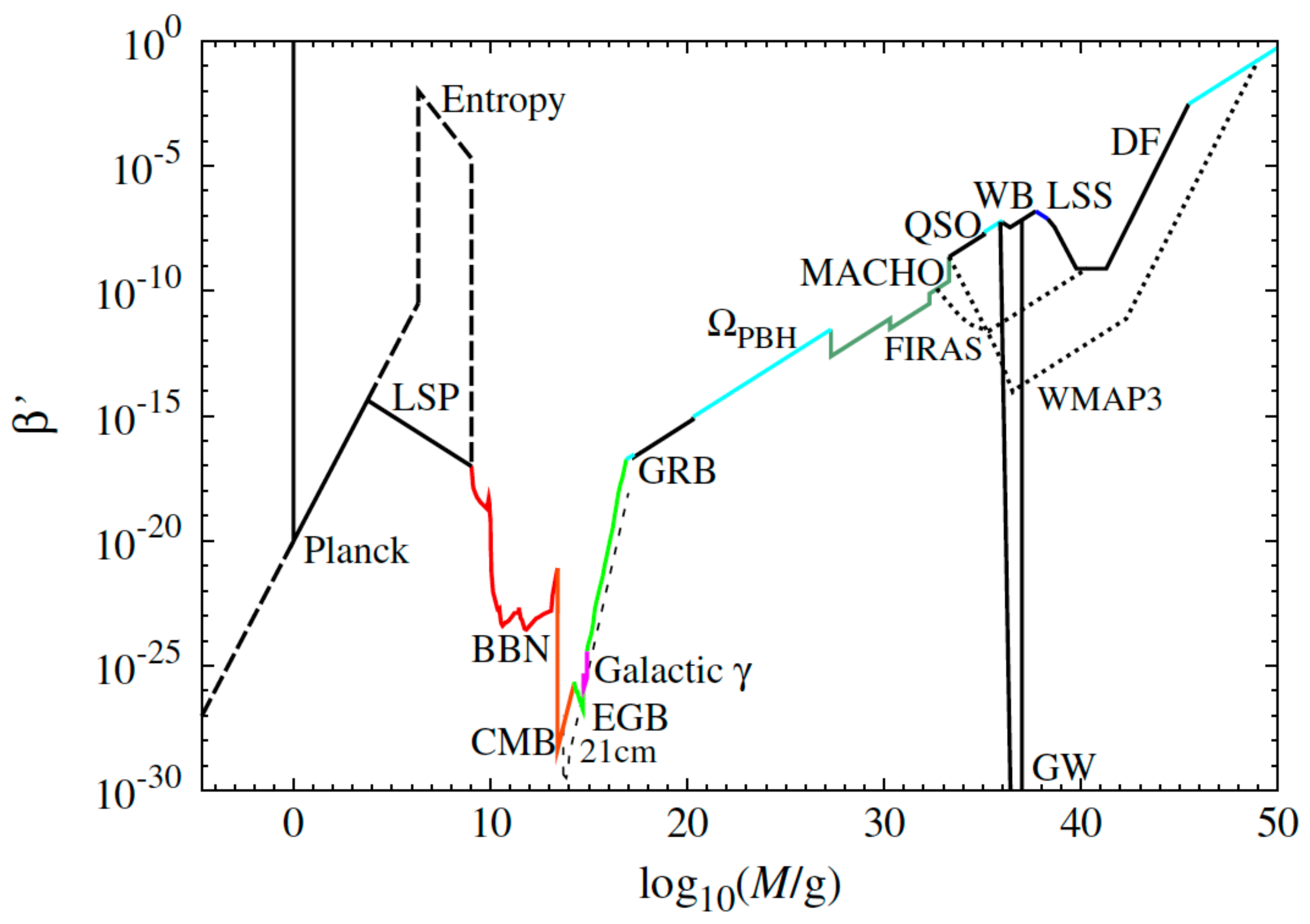

Carr et al. 0912.5297 


\section{Primordial Black Holes (PBH)}

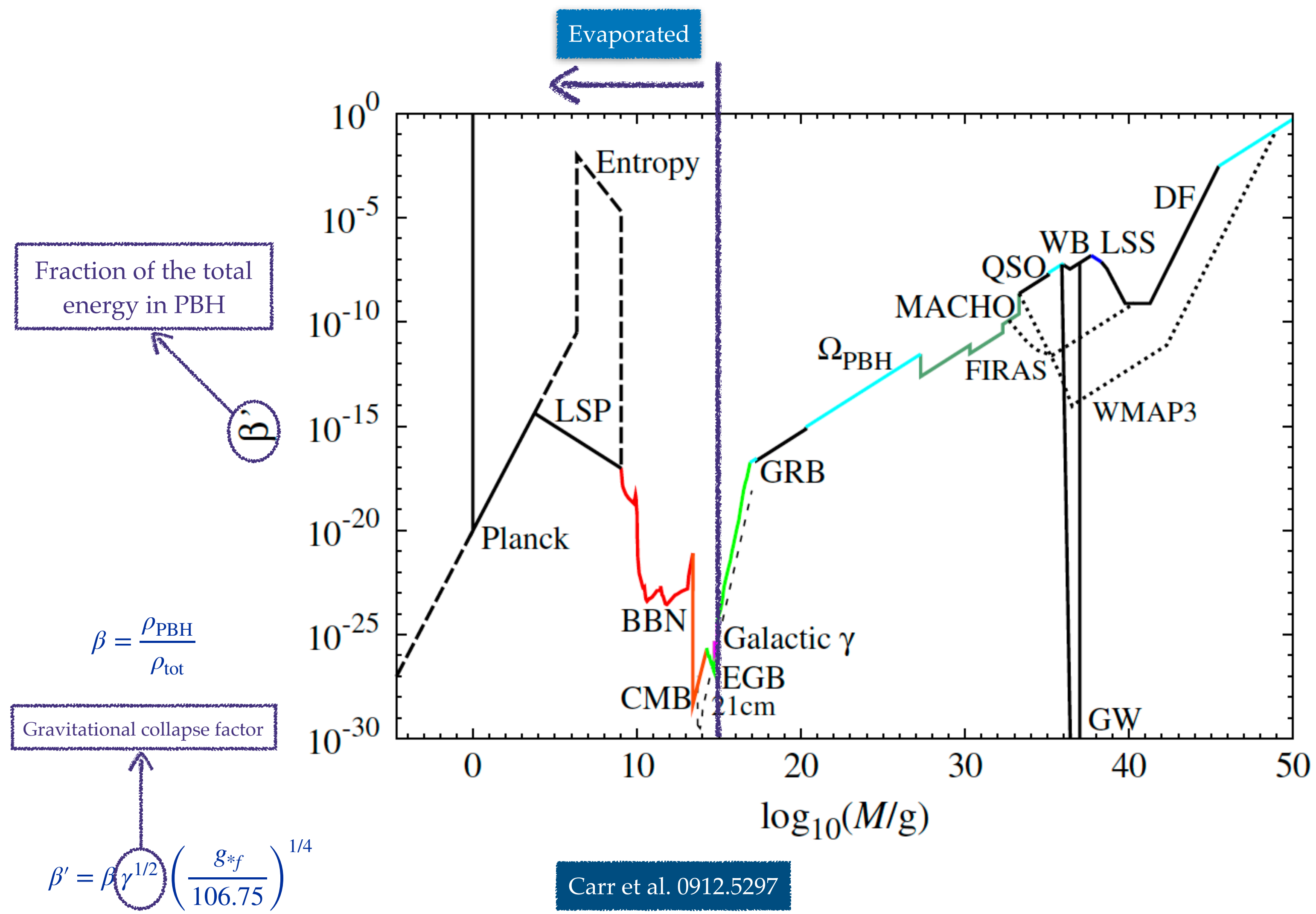




\section{Primordial Black Holes (PBH)}

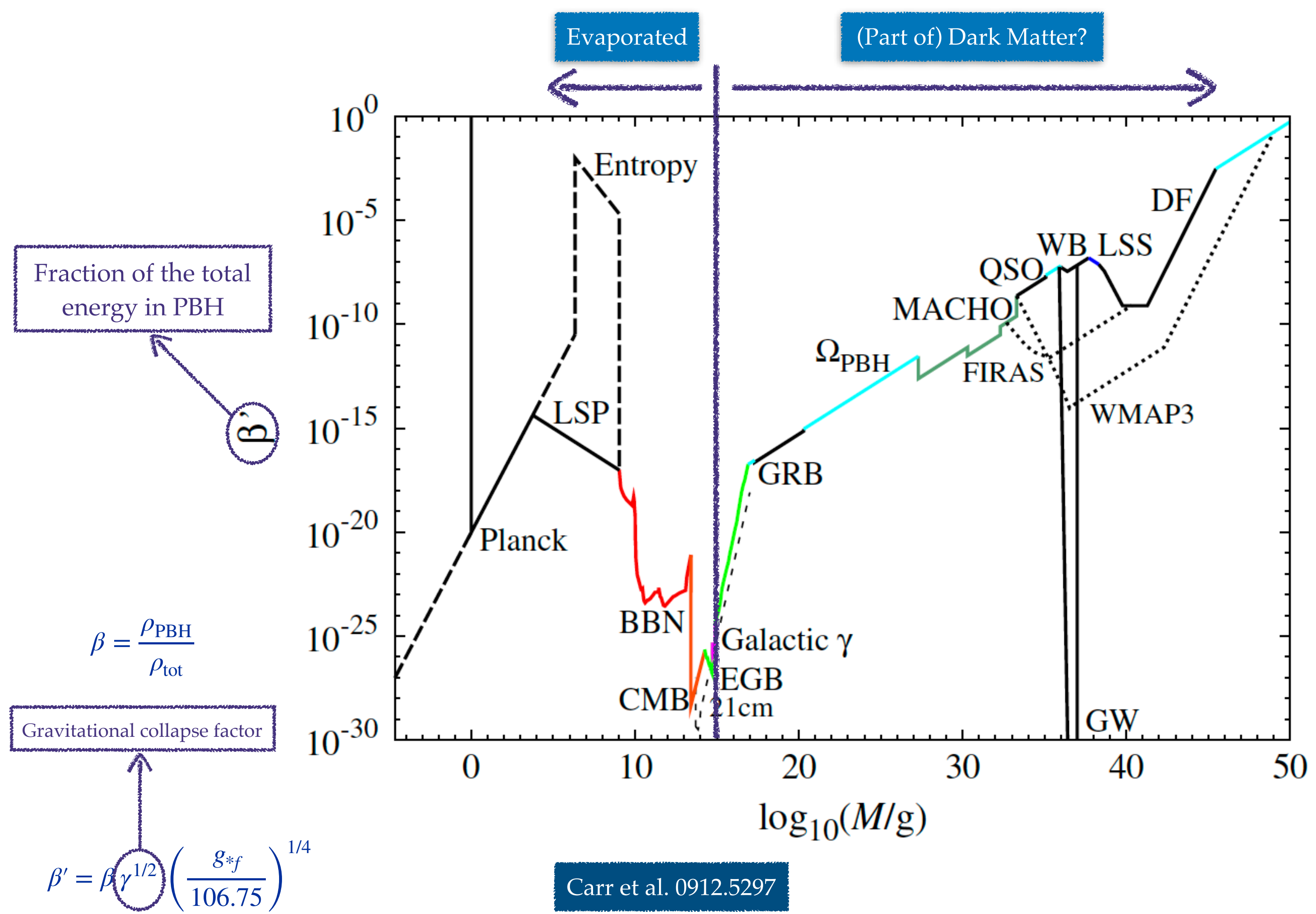




\section{Primordial Black Holes (PBH)}

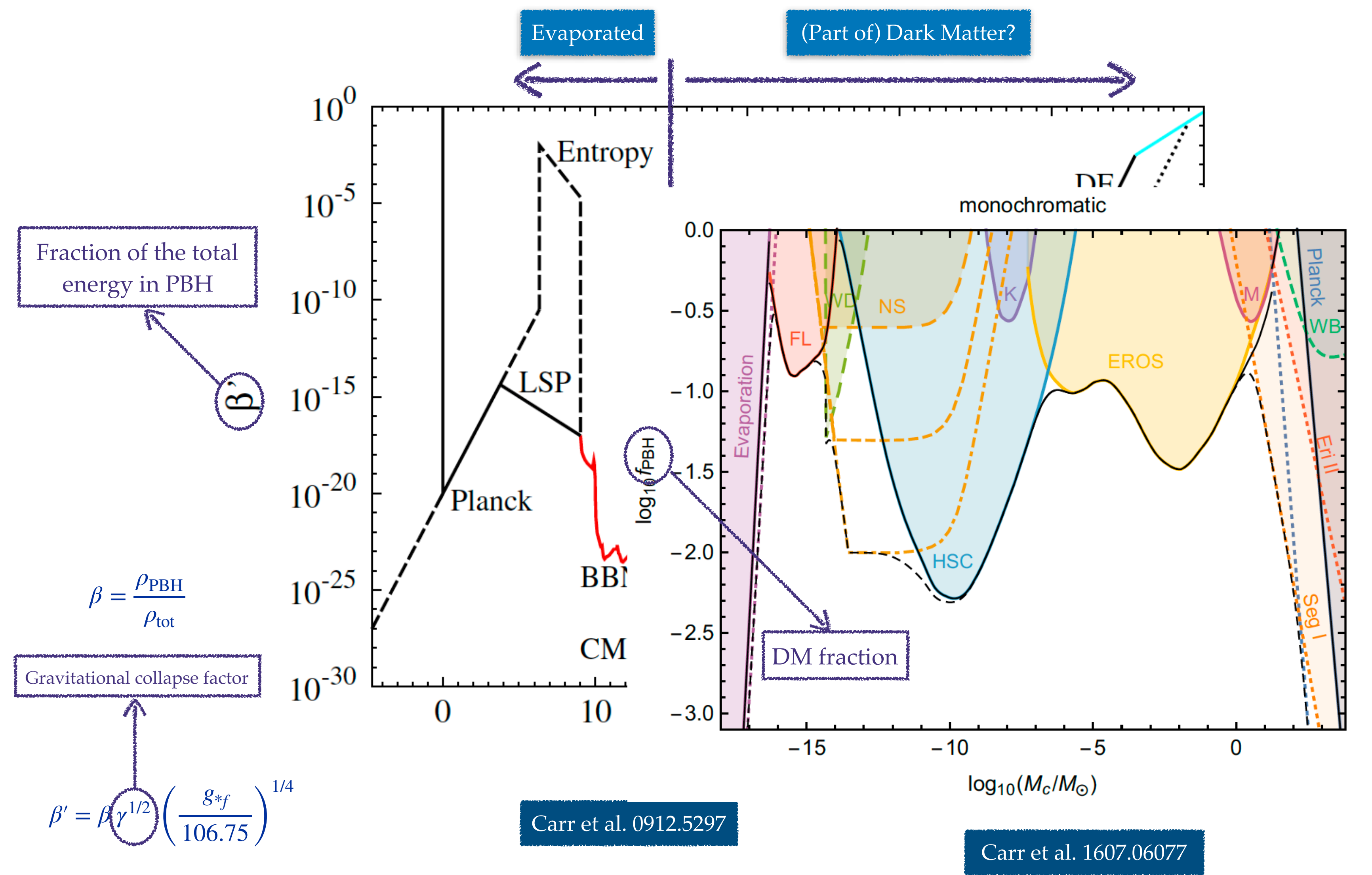


Neutrino emission in the SM

B. Carr, 1976 


\section{Neutrino emission in the SM}

B. Carr, 1976

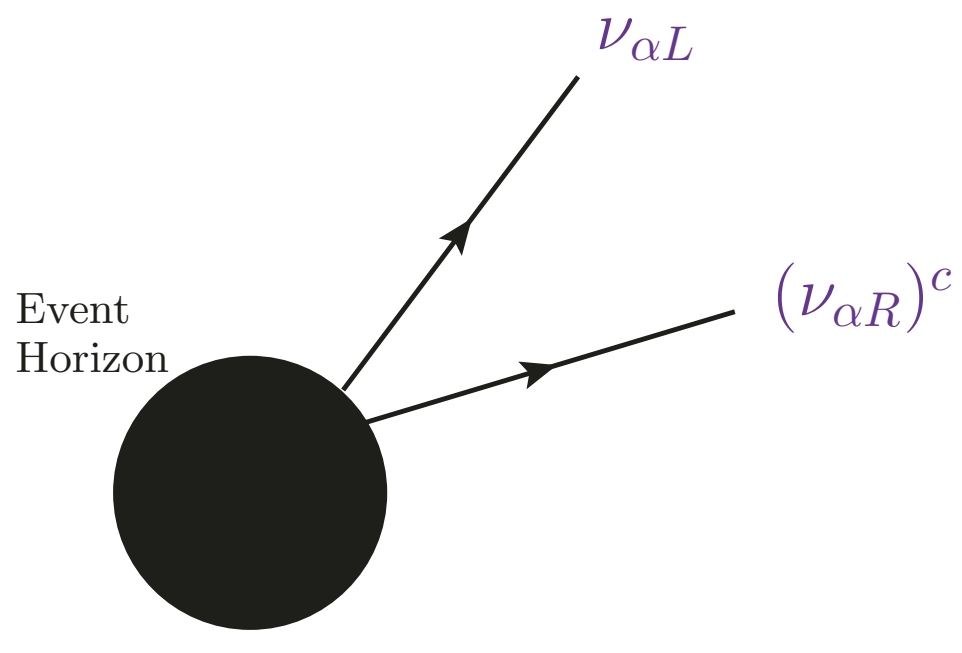

Primary

Component

$$
\alpha=\{e, \mu, \tau\}
$$




\section{Neutrino emission in the SM}

\section{B. Carr, 1976}

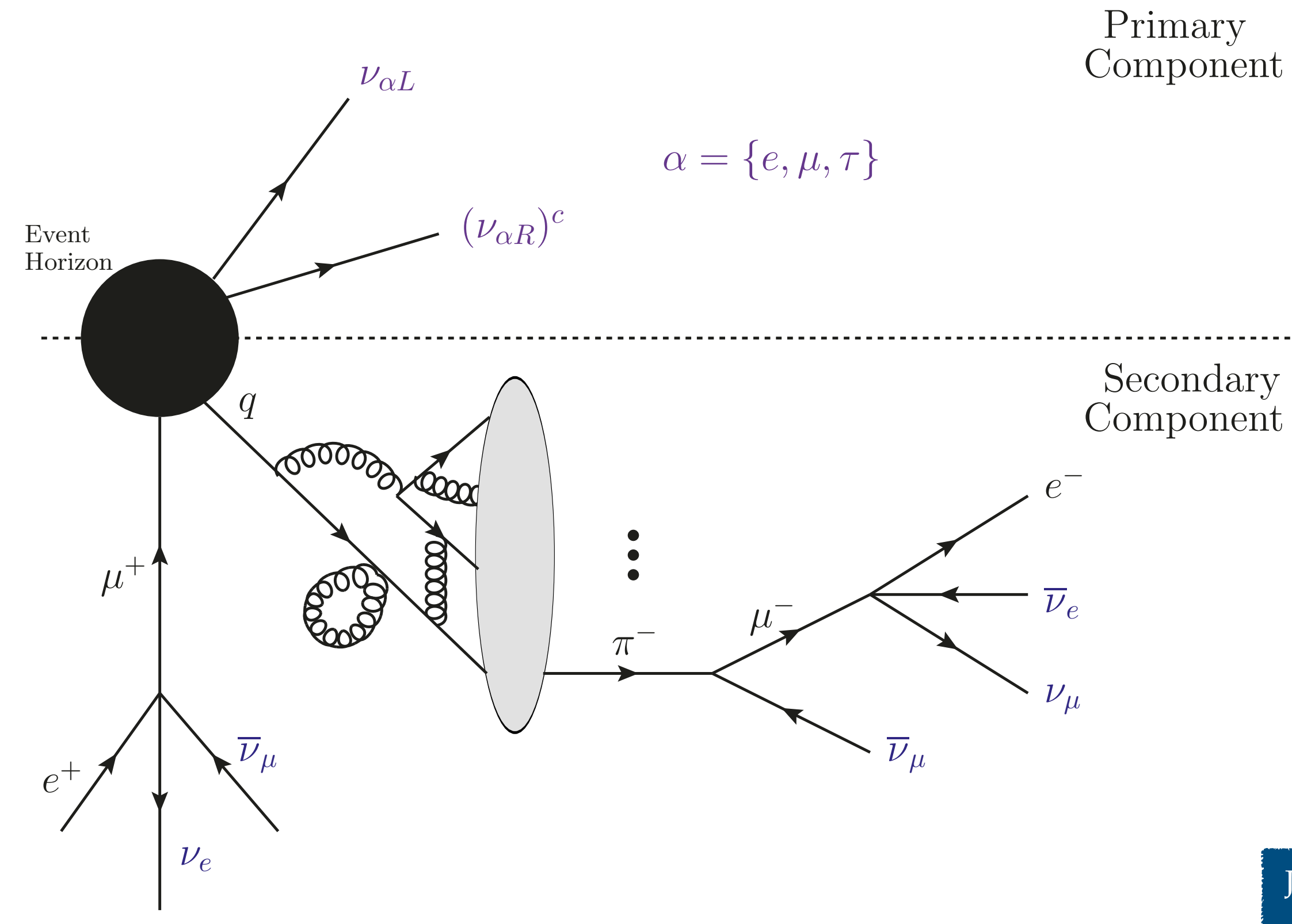

Constraints on the diffuse neutrino flux 


\section{What is the state of the emitted neutrino?}

Neutrinos are

massive

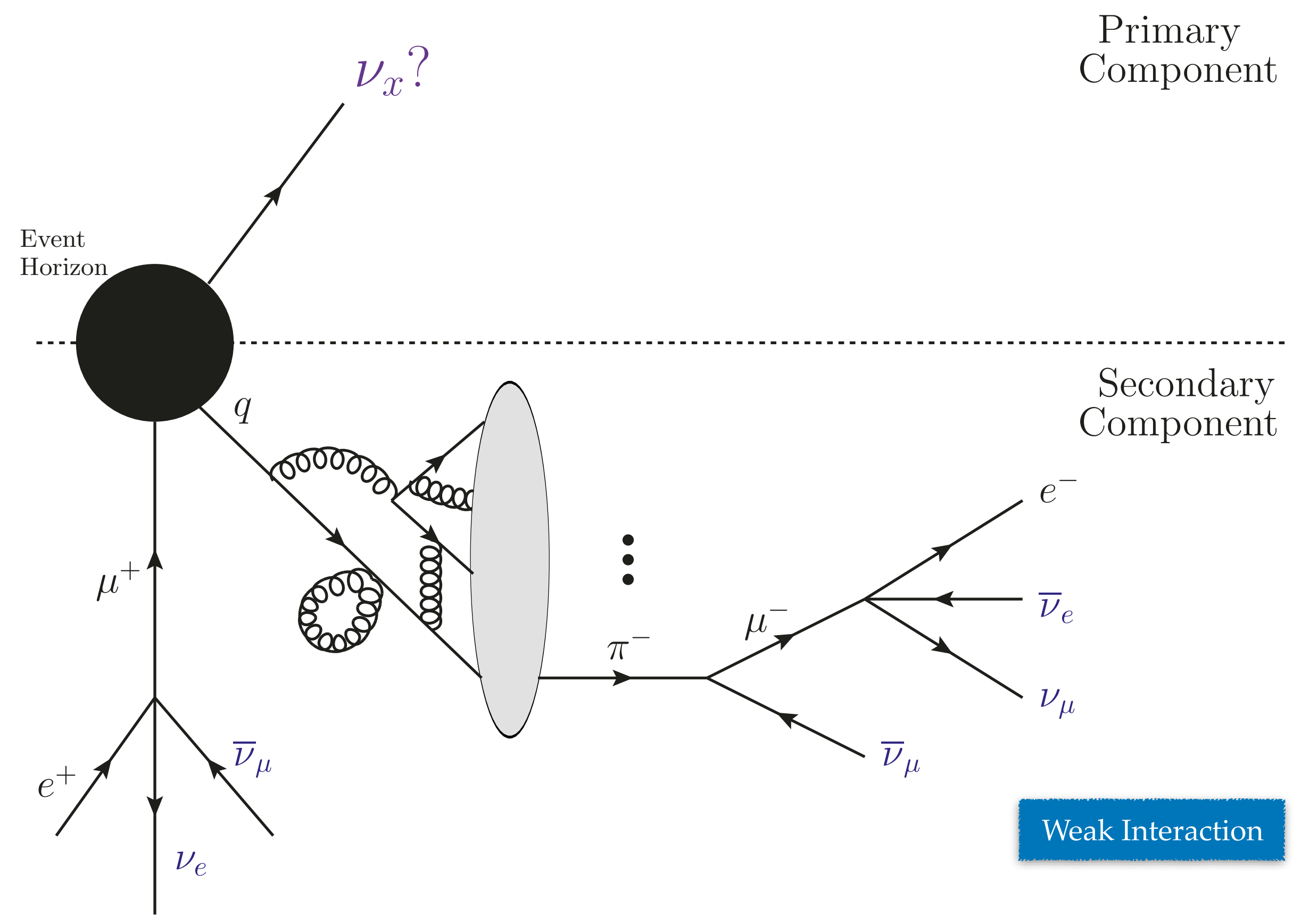




\section{What is the state of the emitted neutrino?}

Weak interactions

Hawking Effect 


\section{What is the state of the emitted neutrino?}

Weak interactions

$n \rightarrow p^{+}+e^{-}+\overline{\nu_{e}}$

Interaction mediated by

a gauge boson

Associated production

of a charged lepton

Flavor eigenstate 


\section{What is the state of the emitted neutrino?}

\section{Weak interactions}

$$
n \rightarrow p^{+}+e^{-}+\overline{\nu_{e}}
$$

Interaction mediated by a gauge boson

Associated production of a charged lepton

Flavor eigenstate

\section{Hawking Effect}

$$
\left\langle 0_{-}\left|b_{i}^{\dagger} b_{i}\right| 0_{-}\right\rangle=\Gamma_{l n}\left[\exp \left(E_{a} / T_{\mathrm{BH}}+1\right)\right]^{-1}
$$

Particle definition in a curved spacetime is observer dependent

No associated production of a charged lepton

Mass eigenstate 


\section{Neutrino instantaneous spectrum}

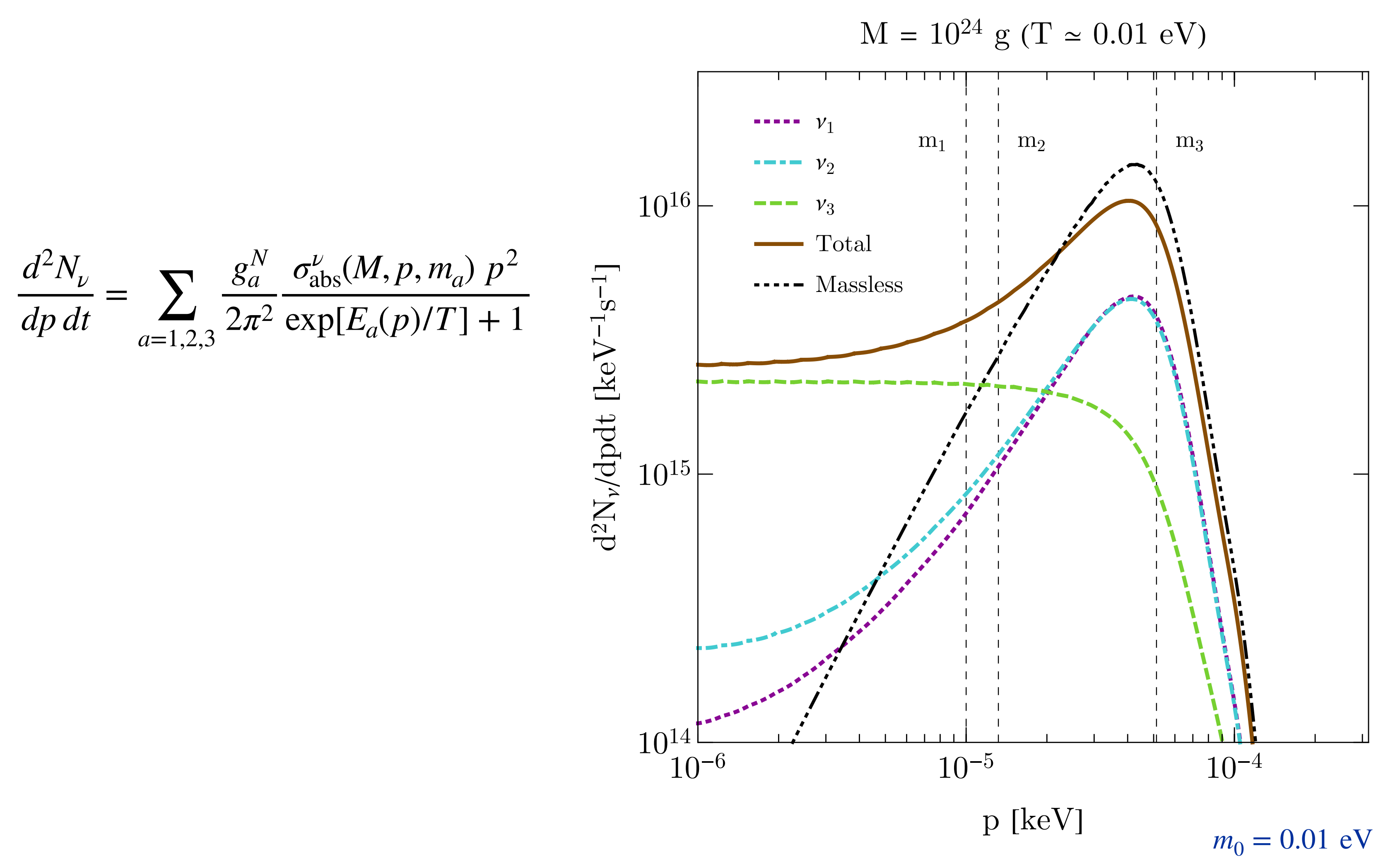




\section{Neutrino instantaneous spectrum}
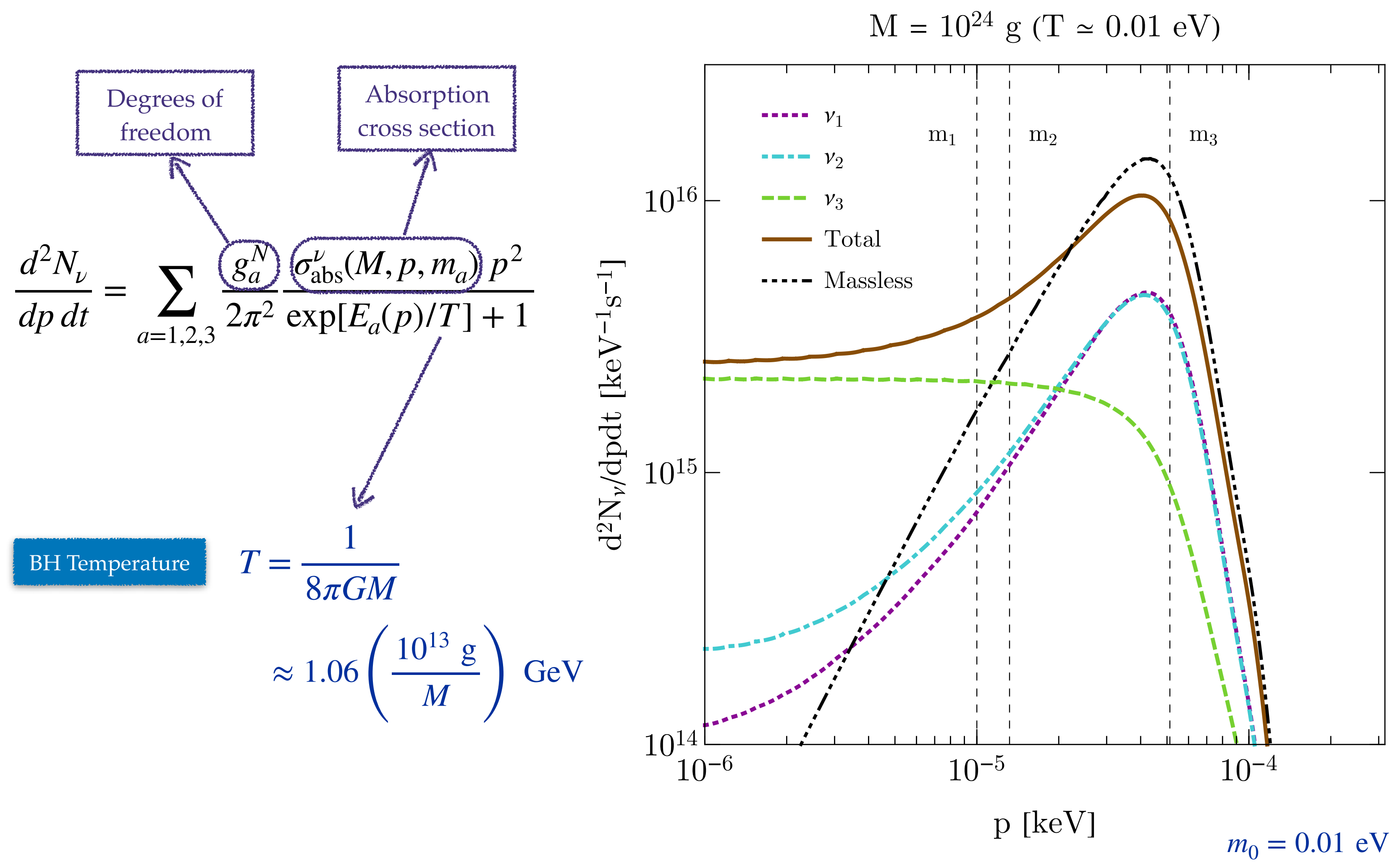


\section{Dirac vs Majorana}




\section{Dirac vs Majorana}

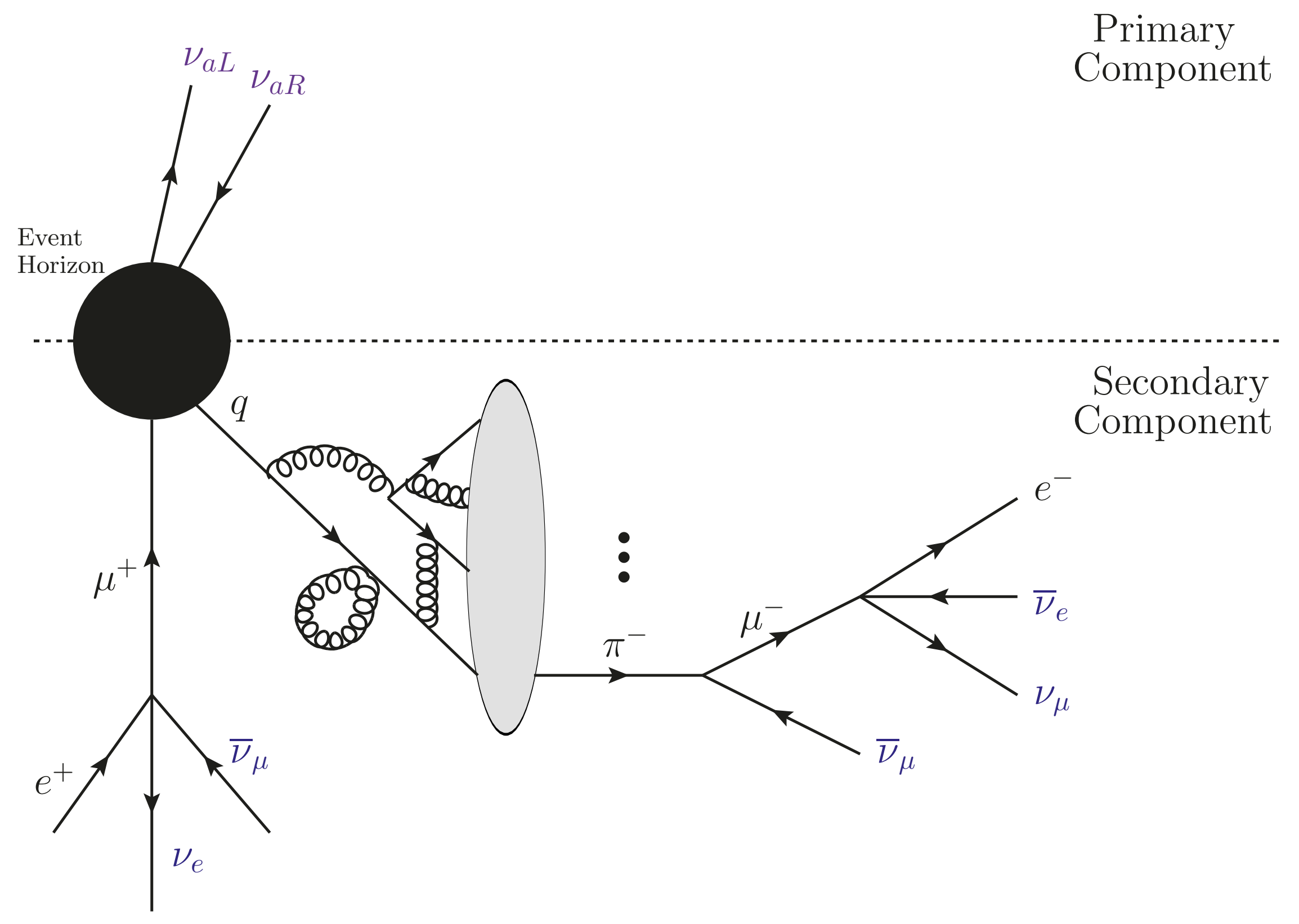

\section{Majorana neutrinos}




\section{Dirac vs Majorana}

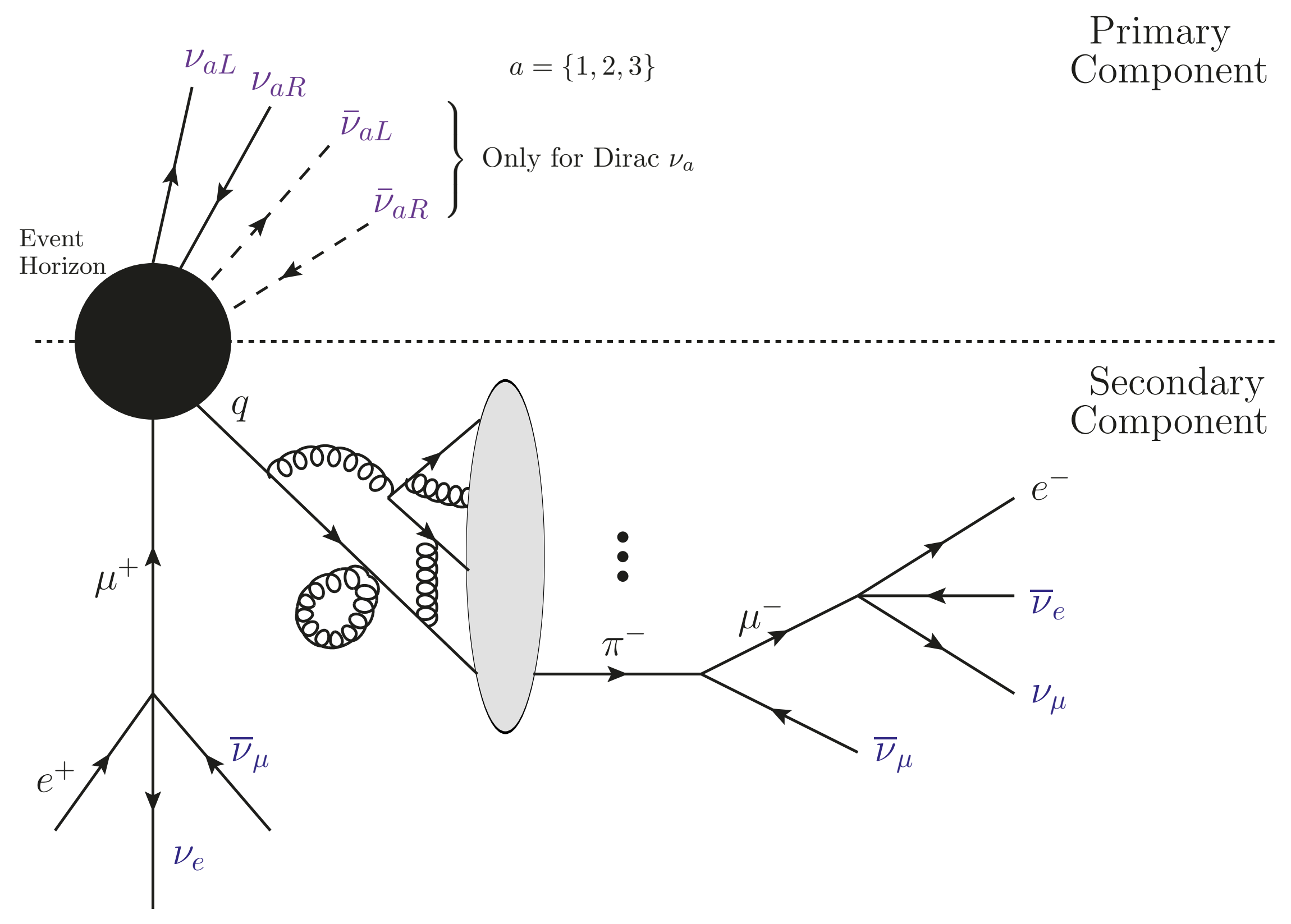

\section{Dirac neutrinos}




\section{Dirac vs Majorana}

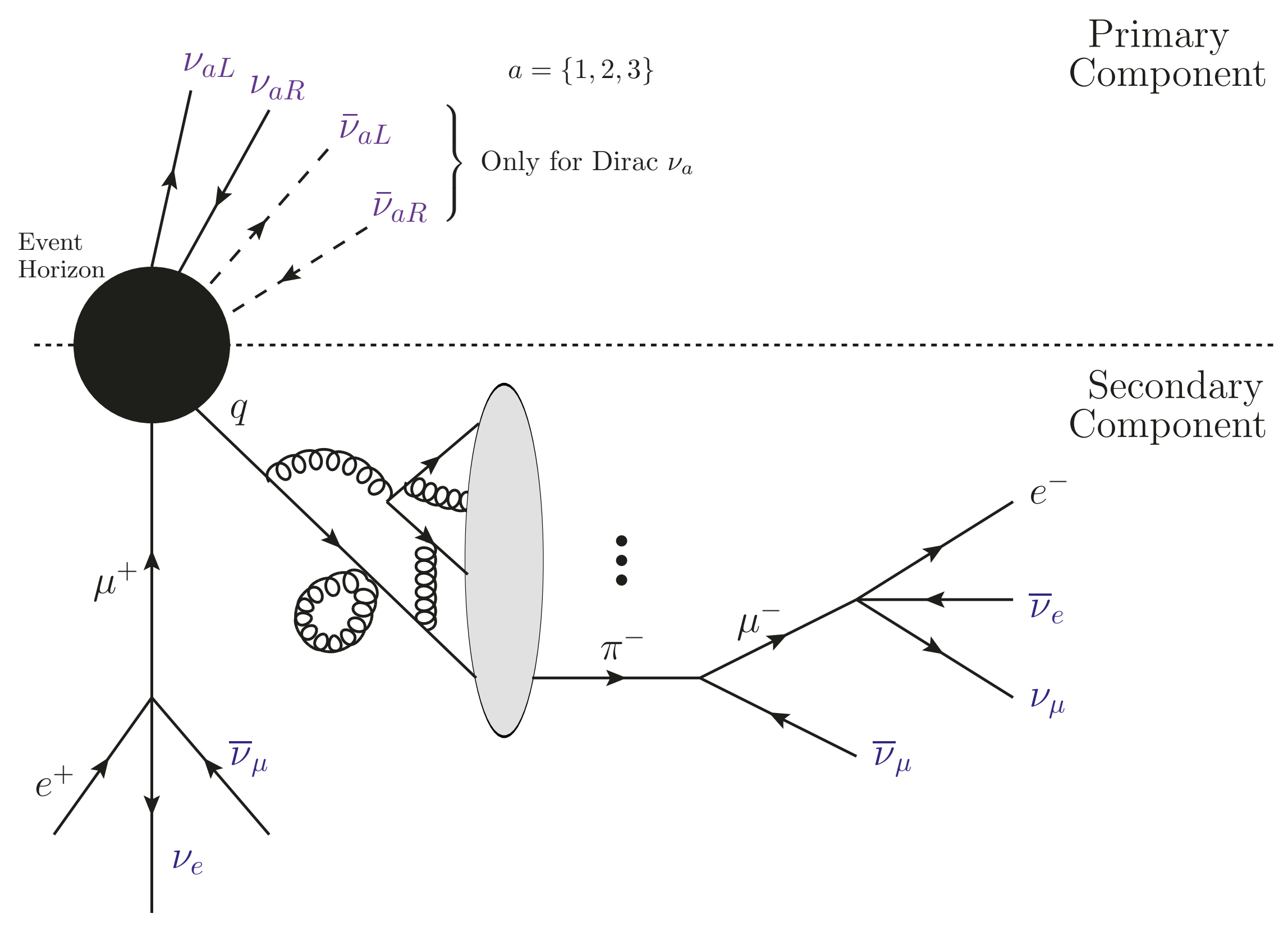

Dirac neutrinos 


\section{Dirac vs Majorana}

Dirac neutrinos

Majorana neutrinos 


\section{Dirac vs Majorana}

Dirac neutrinos 


\section{Dirac vs Majorana}

Dirac neutrinos 


\section{Dirac vs Majorana}

\section{Dirac neutrinos}

Majorana neutrinos

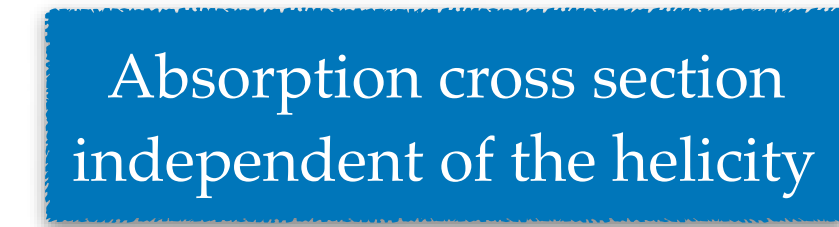

$$
\sigma_{\mathrm{abs}}^{\nu}(+1 / 2)=\sigma_{\mathrm{abs}}^{\nu}(-1 / 2)
$$

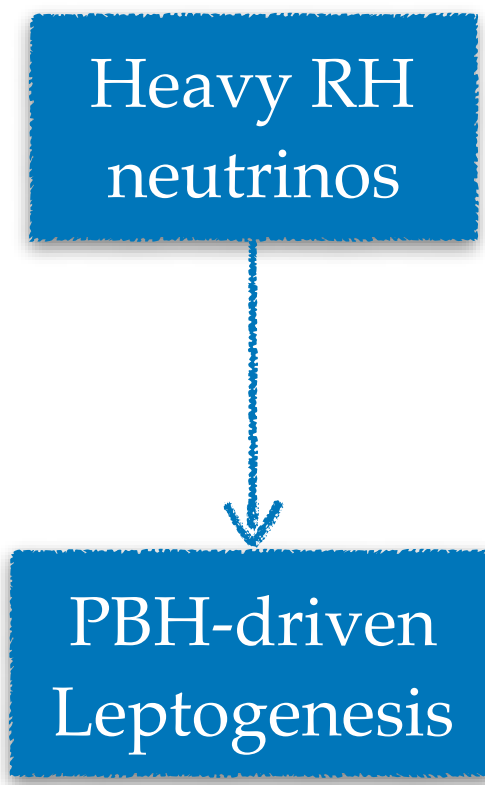

Yamada and Iso, 1610.02586

Morrison et al,1812.10606

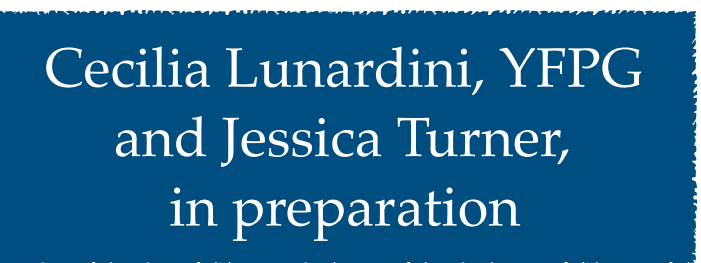




\section{Dirac vs Majorana}

\section{Dirac neutrinos}

Majorana neutrinos

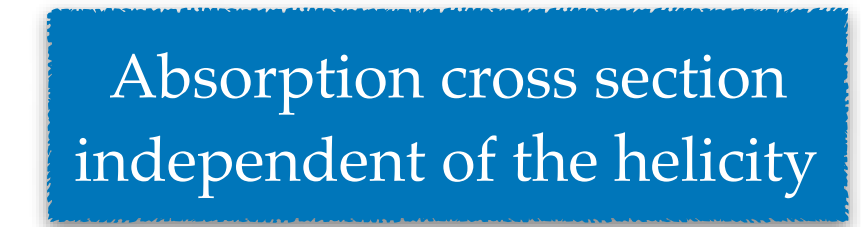

$$
\sigma_{\mathrm{abs}}^{\nu}(+1 / 2)=\sigma_{\mathrm{abs}}^{\nu}(-1 / 2)
$$

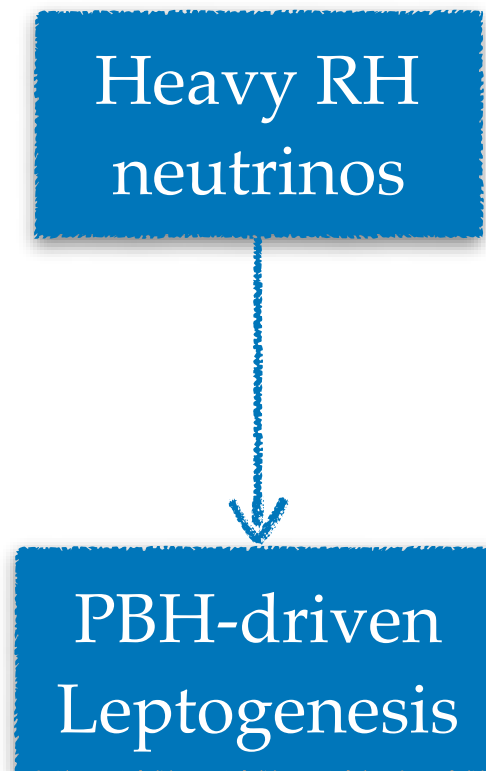

Unruh, 1976

No helicity

suppression

Production of

RH neutrinos!

Yamada and Iso, 1610.02586

Morrison et al,1812.10606

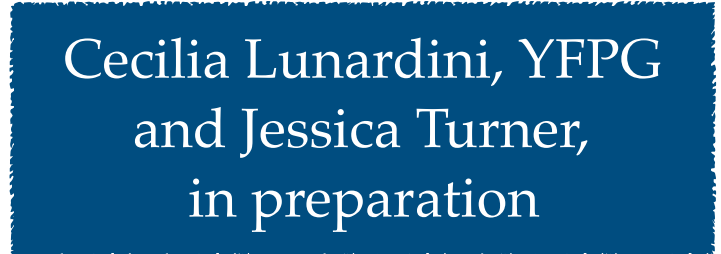




\section{Constraints in the Dirac neutrino case}




\section{Constraints in the Dirac neutrino case}

Let us consider the minimal extension

$$
\mathscr{L}_{Y}=-Y_{\nu}^{a b} \overline{L_{L}^{a}} \widetilde{H} \nu_{b R}
$$




\section{Constraints in the Dirac neutrino case}

Let us consider the minimal extension

$$
\mathscr{L}_{Y}=-Y_{\nu}^{a b} \overline{L_{L}^{a}} \widetilde{H} \nu_{b R}
$$

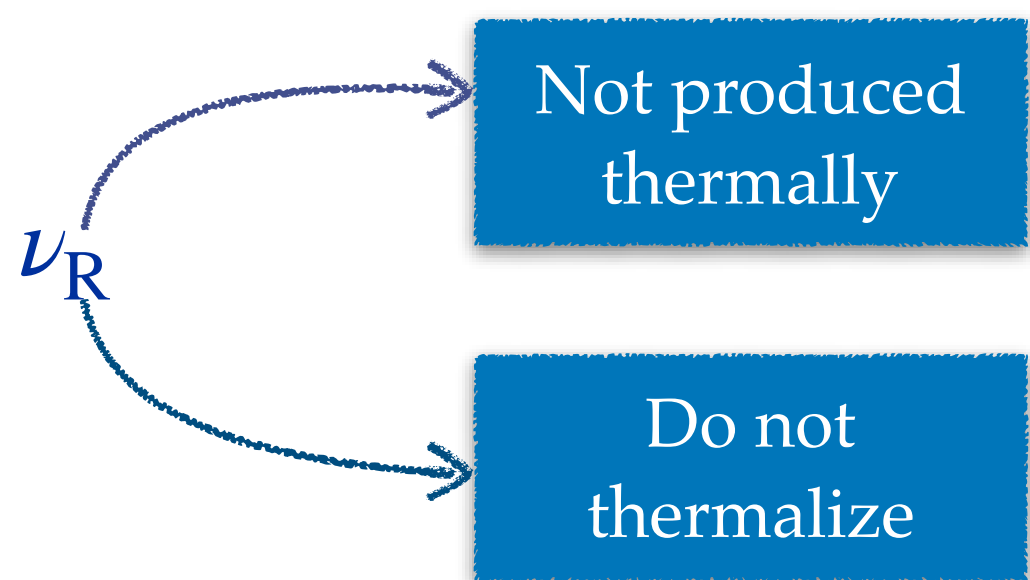

Antonelli et al, 1981

Chen et al, 1509.00481 


\section{Constraints in the Dirac neutrino case}

Let us consider the

minimal extension

$$
\mathscr{L}_{Y}=-Y_{\nu}^{a b} \overline{L_{L}^{a}} \widetilde{H} \nu_{b R}
$$

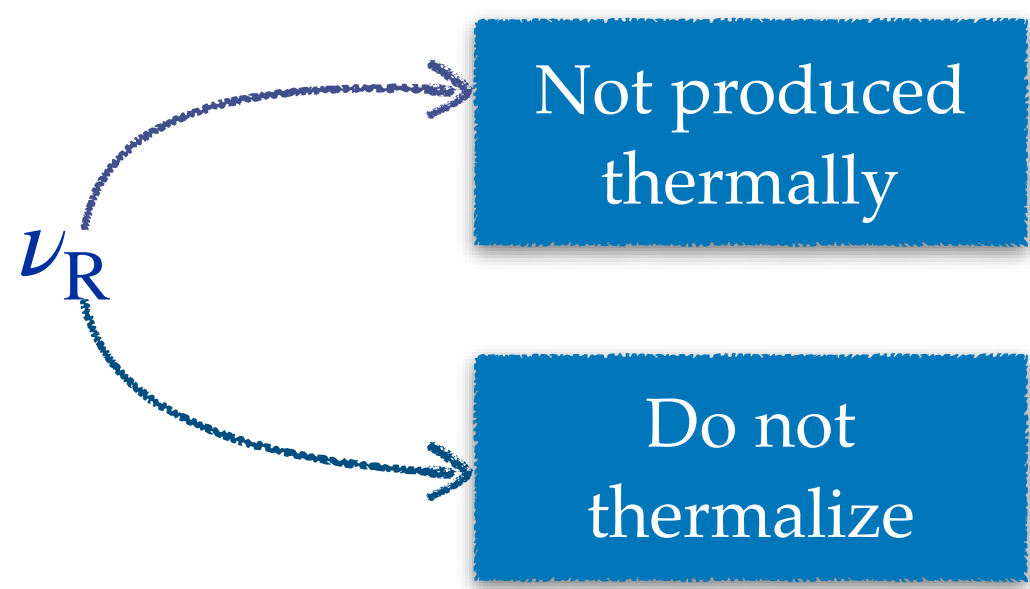

\section{PBHs are}

formed

$$
t_{i}, T_{\mathrm{f}}
$$




\section{Constraints in the Dirac neutrino case}

Let us consider the

minimal extension

$$
\mathscr{L}_{Y}=-Y_{\nu}^{a b} \overline{L_{L}^{a}} \widetilde{H} \nu_{b R}
$$

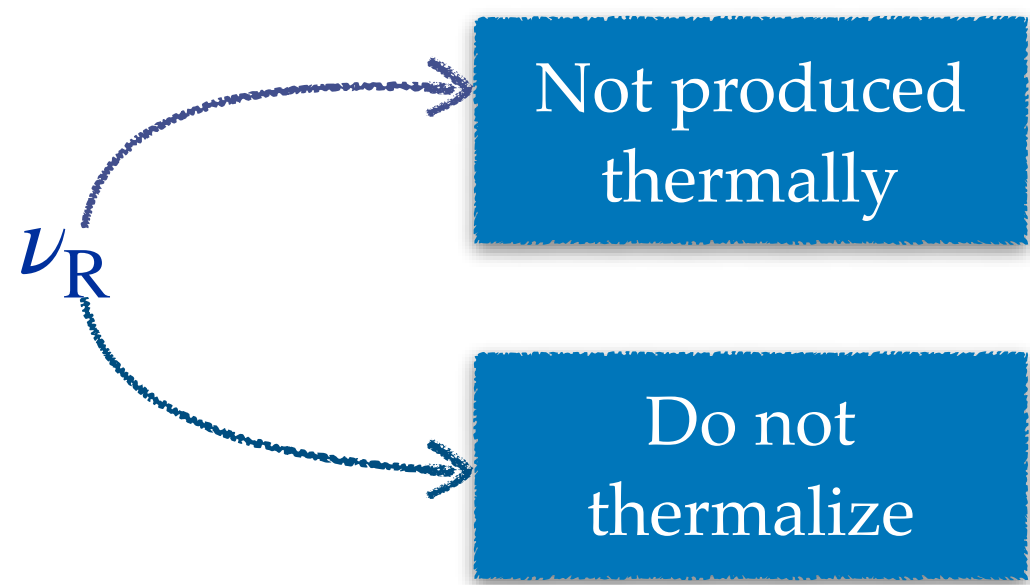

Antonelli et al, 1981

Chen et al, 1509.00481

\section{PBHs are formed \\ PBHs \\ evaporate \\ $t_{i}, T_{\mathrm{f}}$ \\ $t_{\mathrm{EV}}, T_{\mathrm{EV}}$}




\section{Constraints in the Dirac neutrino case}

\section{Let us consider the minimal extension}

$$
\mathscr{L}_{Y}=-Y_{\nu}^{a b} \overline{L_{L}^{a}} \widetilde{H} \nu_{b R}
$$

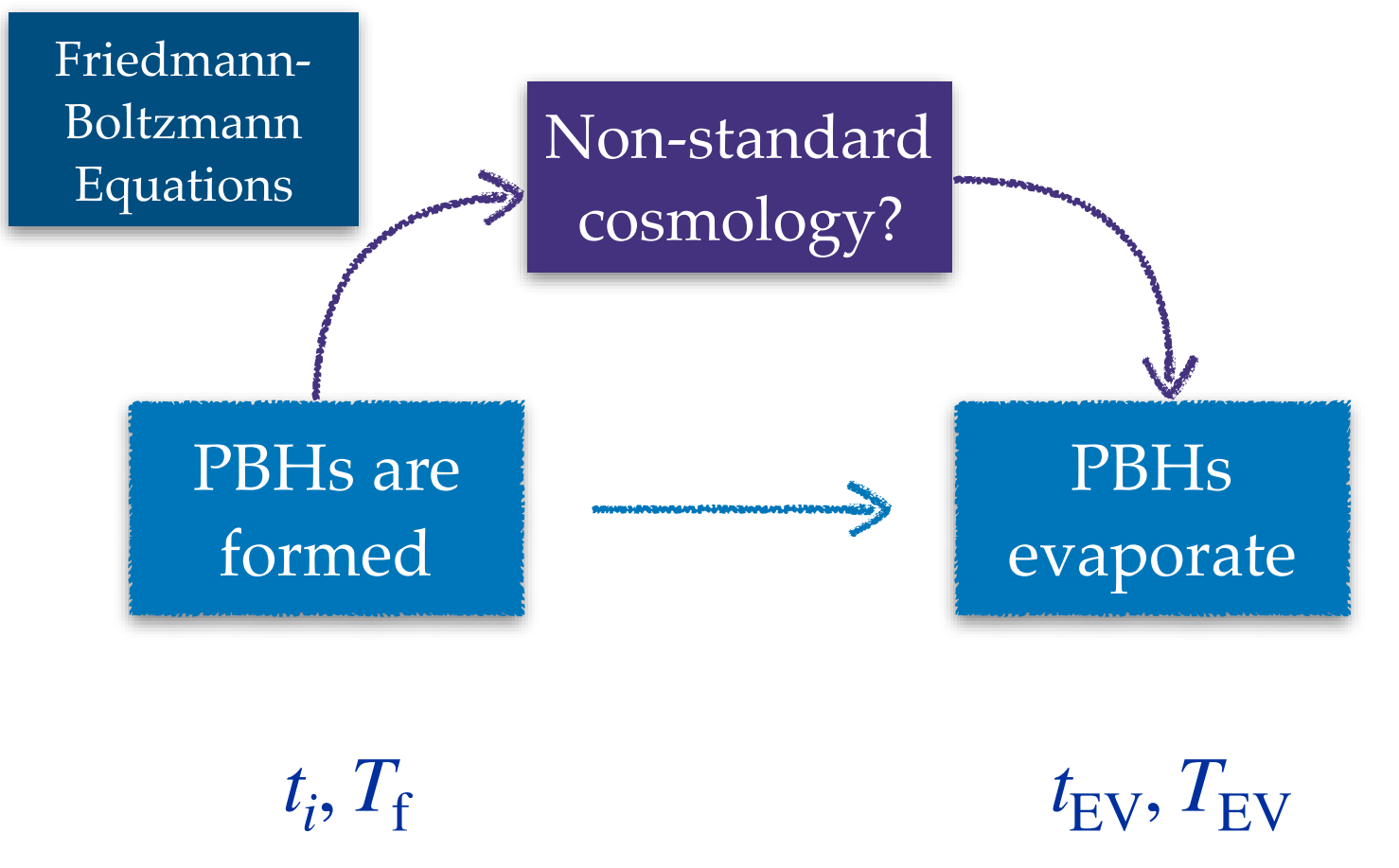

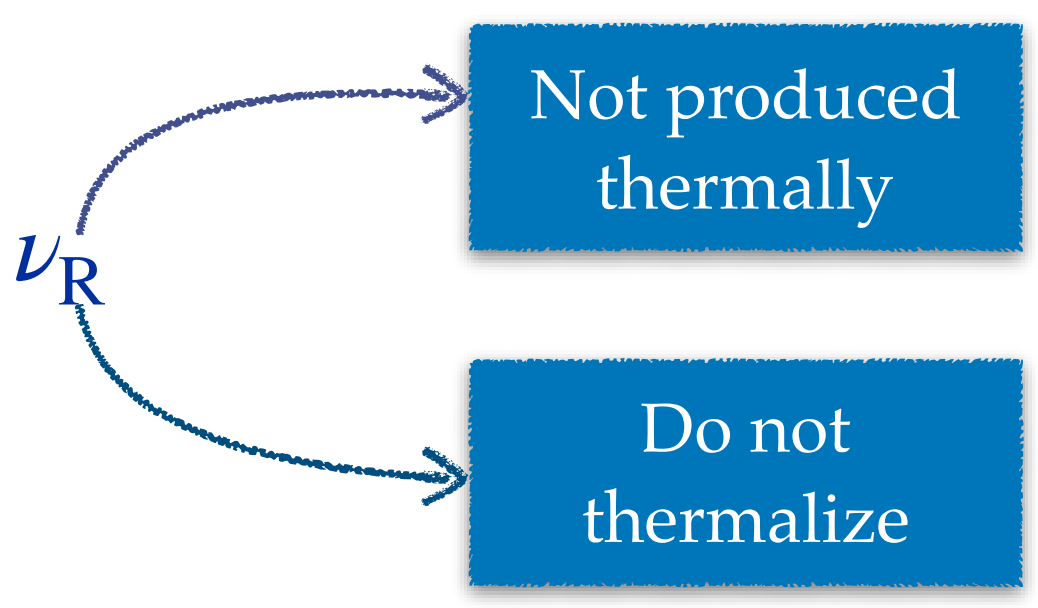

Antonelli et al, 1981

Chen et al, 1509.00481 


\section{Constraints in the Dirac neutrino case}

\section{Let us consider the minimal extension}

$$
\mathscr{L}_{Y}=-Y_{\nu}^{a b} \overline{L_{L}^{a}} \widetilde{H} \nu_{b R}
$$

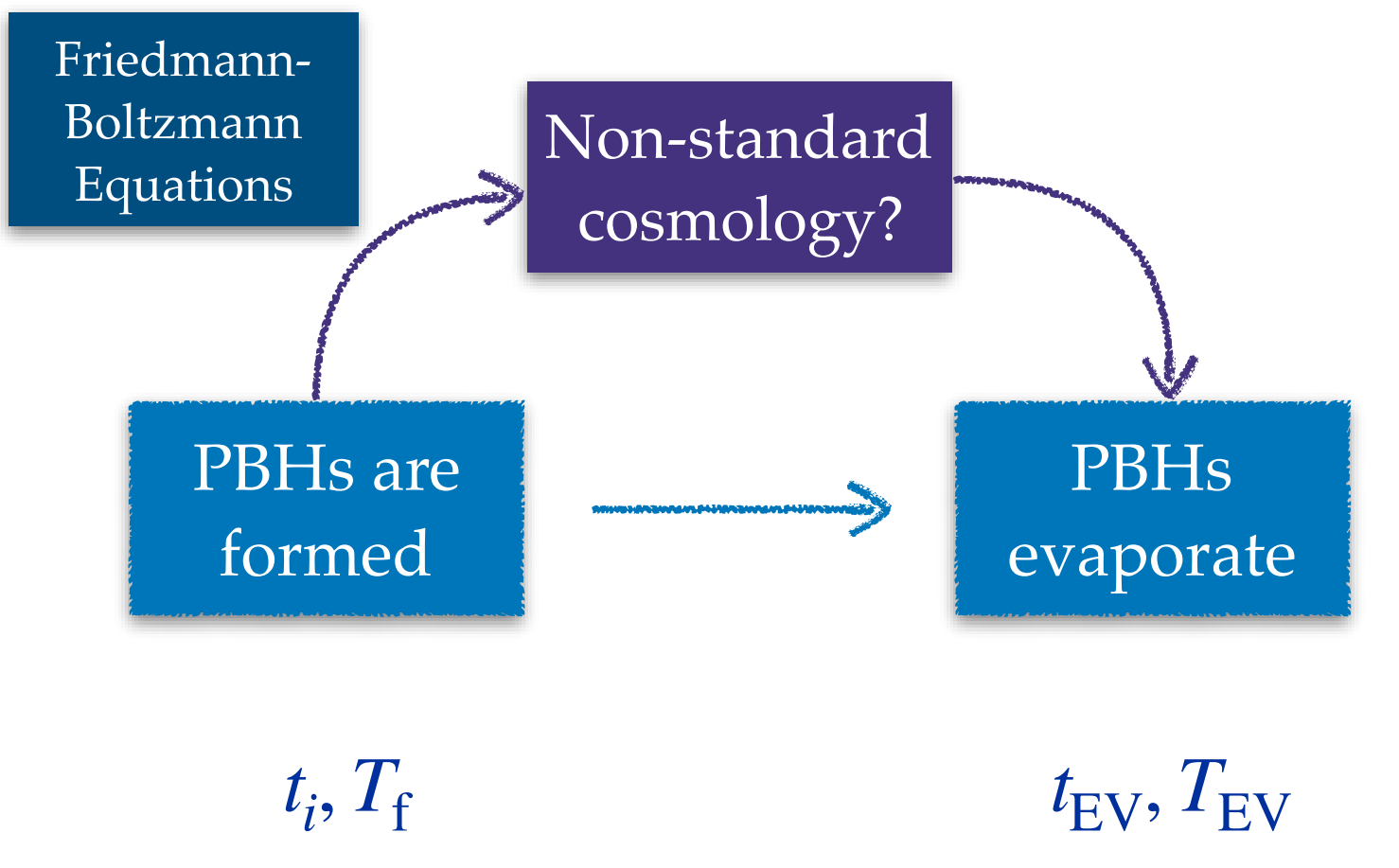

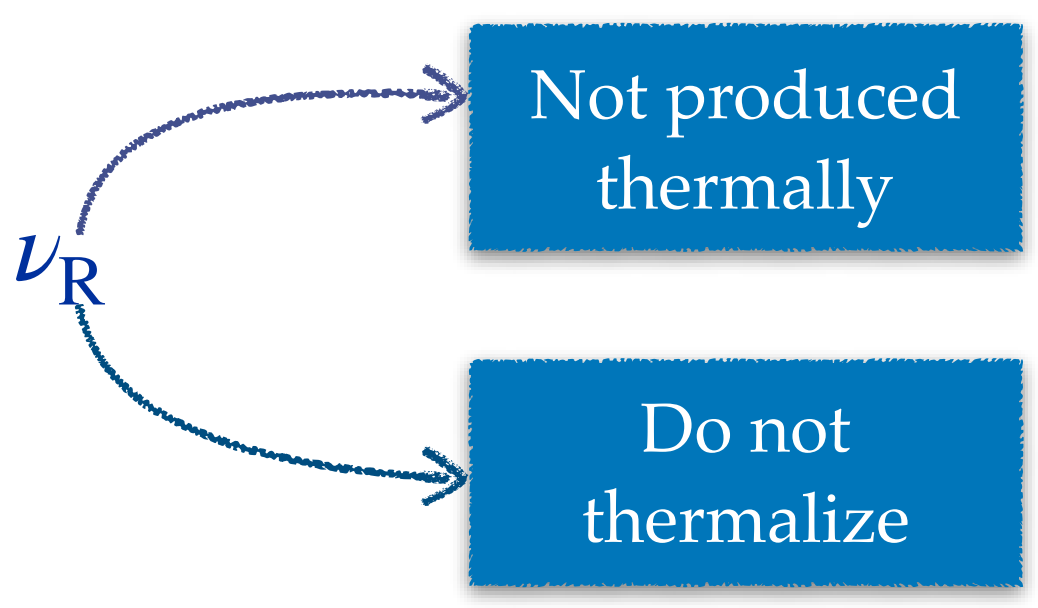

Antonelli et al, 1981

Chen et al, 1509.00481 


\section{Constraints in the Dirac neutrino case}

Let us consider the minimal extension

$$
\mathscr{L}_{Y}=-Y_{\nu}^{a b} \overline{L_{L}^{a}} \widetilde{H} \nu_{b R}
$$

\section{Friedmann- \\ Boltzmann \\ Equations \\ PBHs are formed}

Non-standard cosmology?

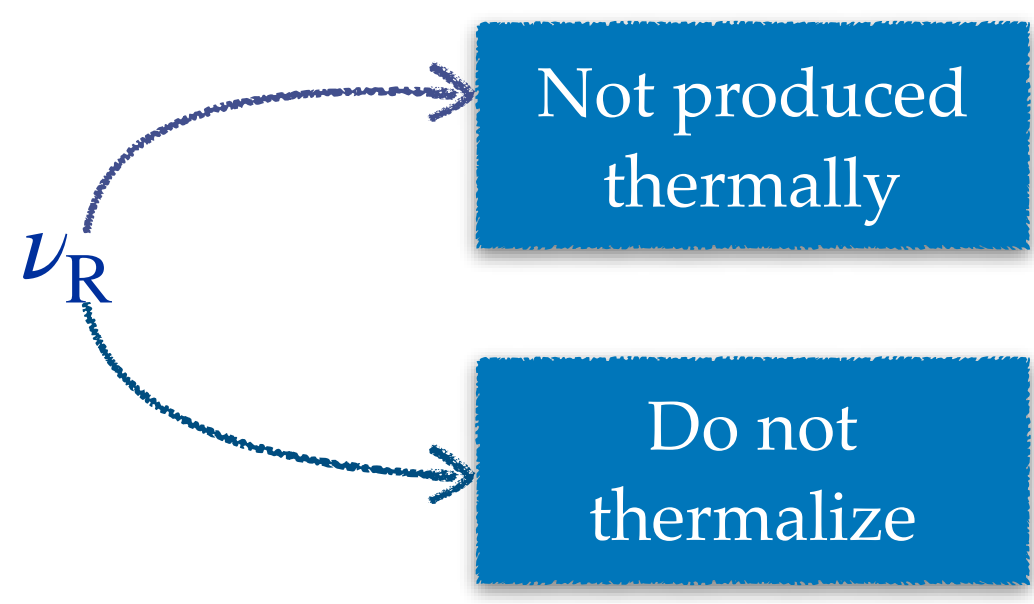

Antonelli et al, 1981

Chen et al, 1509.00481 $t_{i}, T_{\mathrm{f}}$ 


\section{Constraints in the Dirac neutrino case}

Let us consider the

minimal extension

$$
\mathscr{L}_{Y}=-Y_{\nu}^{a b} \overline{L_{L}^{a}} \widetilde{H} \nu_{b R}
$$

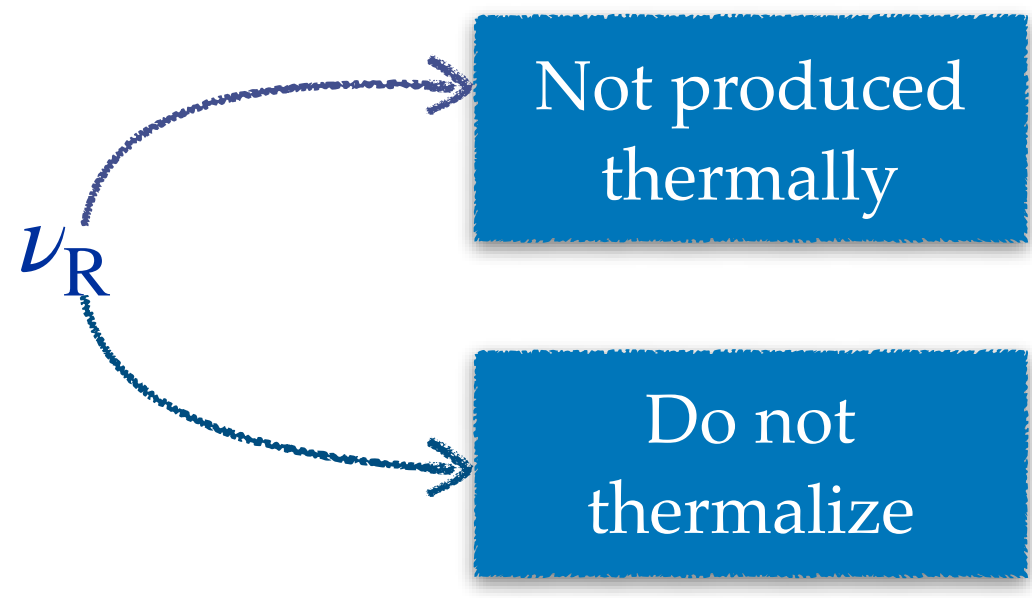

Antonelli et al, 1981

Chen et al, 1509.00481

$$
\begin{aligned}
& \text { Friedmann- } \\
& t_{i}, T_{\mathrm{f}} \\
& t_{\mathrm{EV}}, T_{\mathrm{EV}} \\
& \Delta N_{\mathrm{eff}}=\left\{\frac{8}{7}\left(\frac{4}{11}\right)^{-\frac{4}{3}}+N_{\mathrm{eff}}^{\mathrm{SM}}\right\} \frac{\rho_{\nu_{\mathrm{R}}}\left(T_{\mathrm{EV}}\right)}{\rho_{\mathrm{R}}\left(T_{\mathrm{EV}}\right)}\left(\frac{g_{*}\left(T_{\mathrm{EV}}\right)}{g_{*}\left(T_{\mathrm{EQ}}\right)}\right)\left(\frac{g_{* S}\left(T_{\mathrm{EQ}}\right)}{g_{* S}\left(T_{\mathrm{EV}}\right)}\right)^{\frac{4}{3}}
\end{aligned}
$$

\section{Matter-}




\section{Constraints in the Dirac neutrino case}

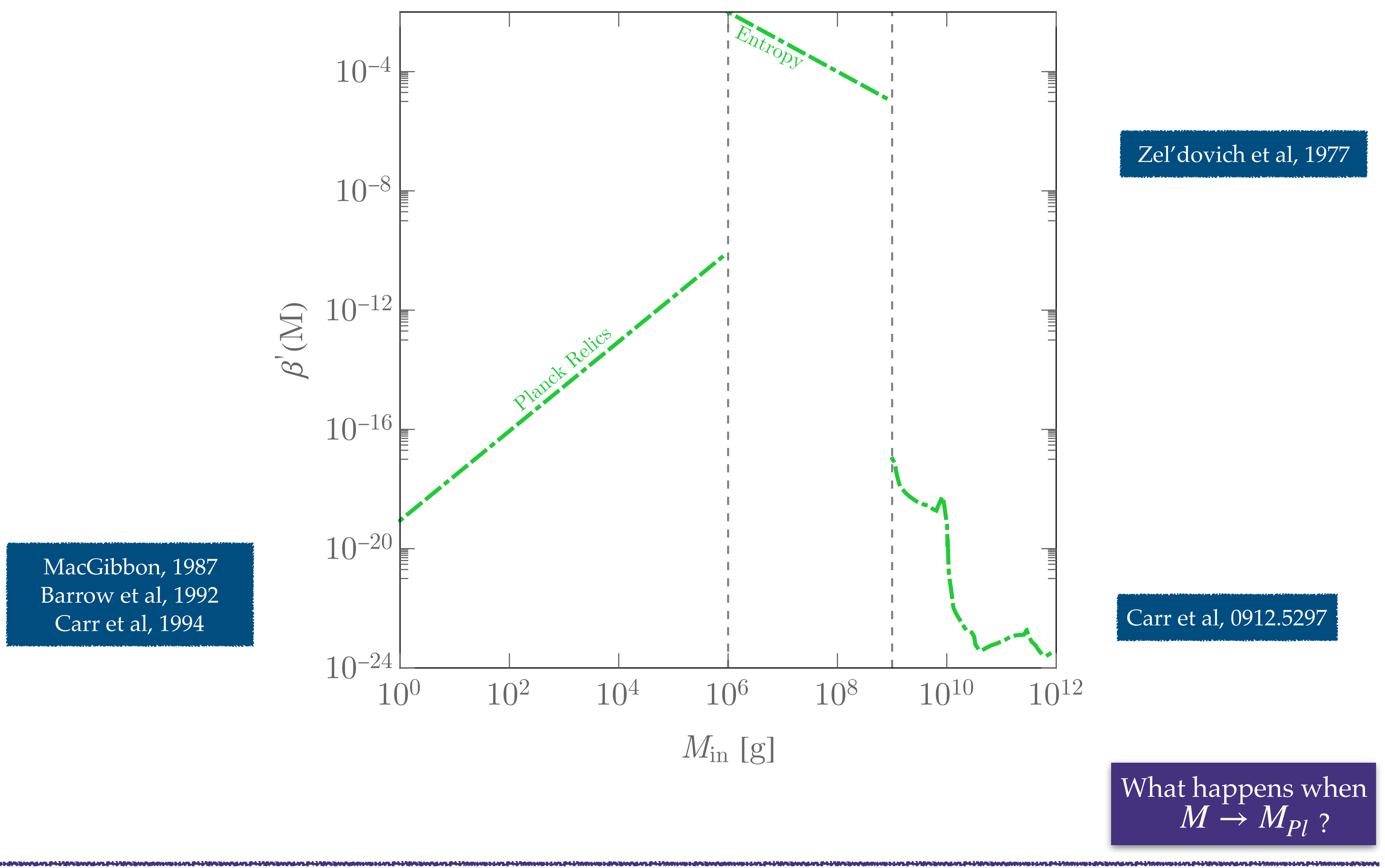




\section{Constraints in the Dirac neutrino case}

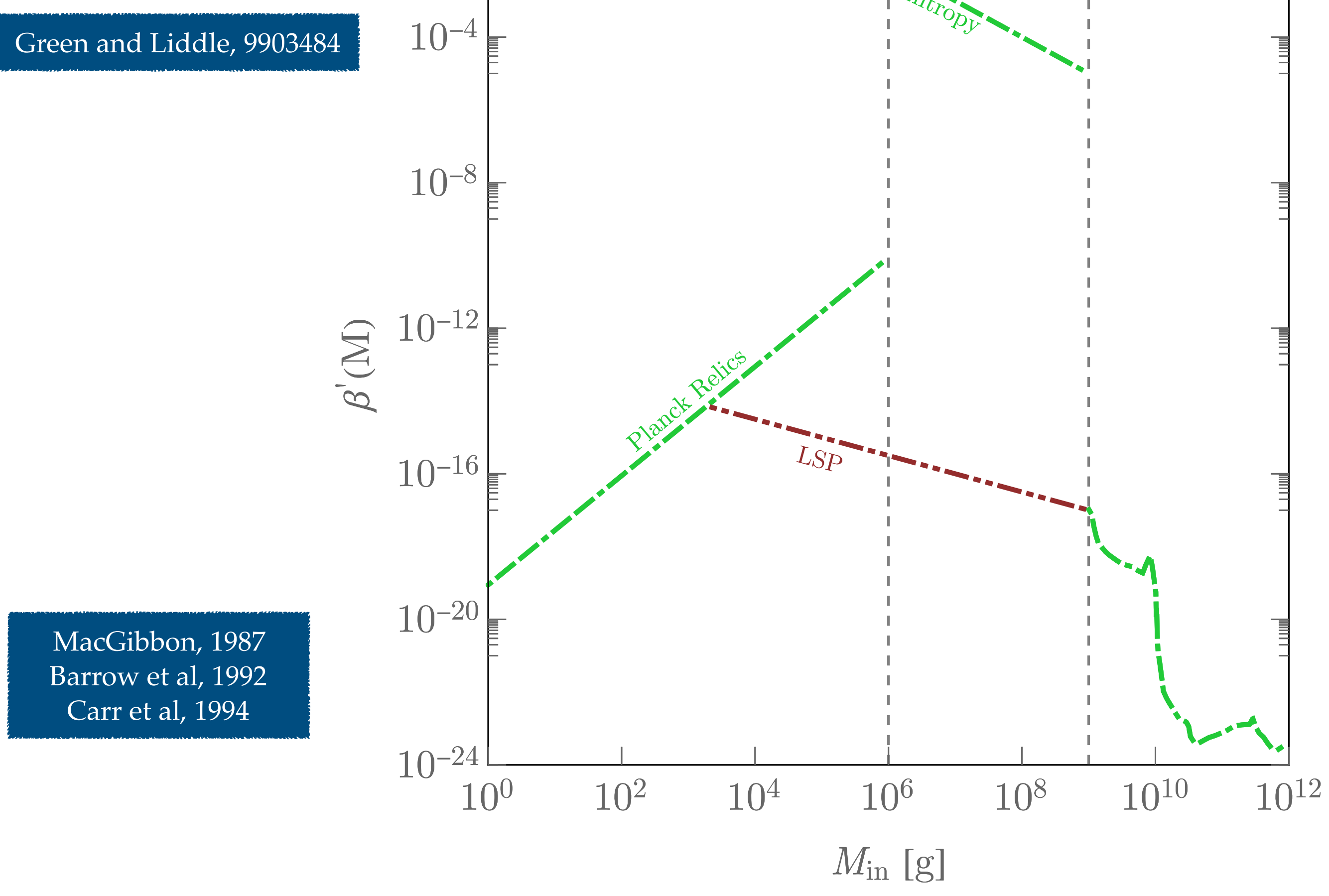

Zel'dovich et al, 1977

Carr et al, 0912.5297

What happens when $M \rightarrow M_{P l}$ ? 


\section{Constraints in the Dirac neutrino case}

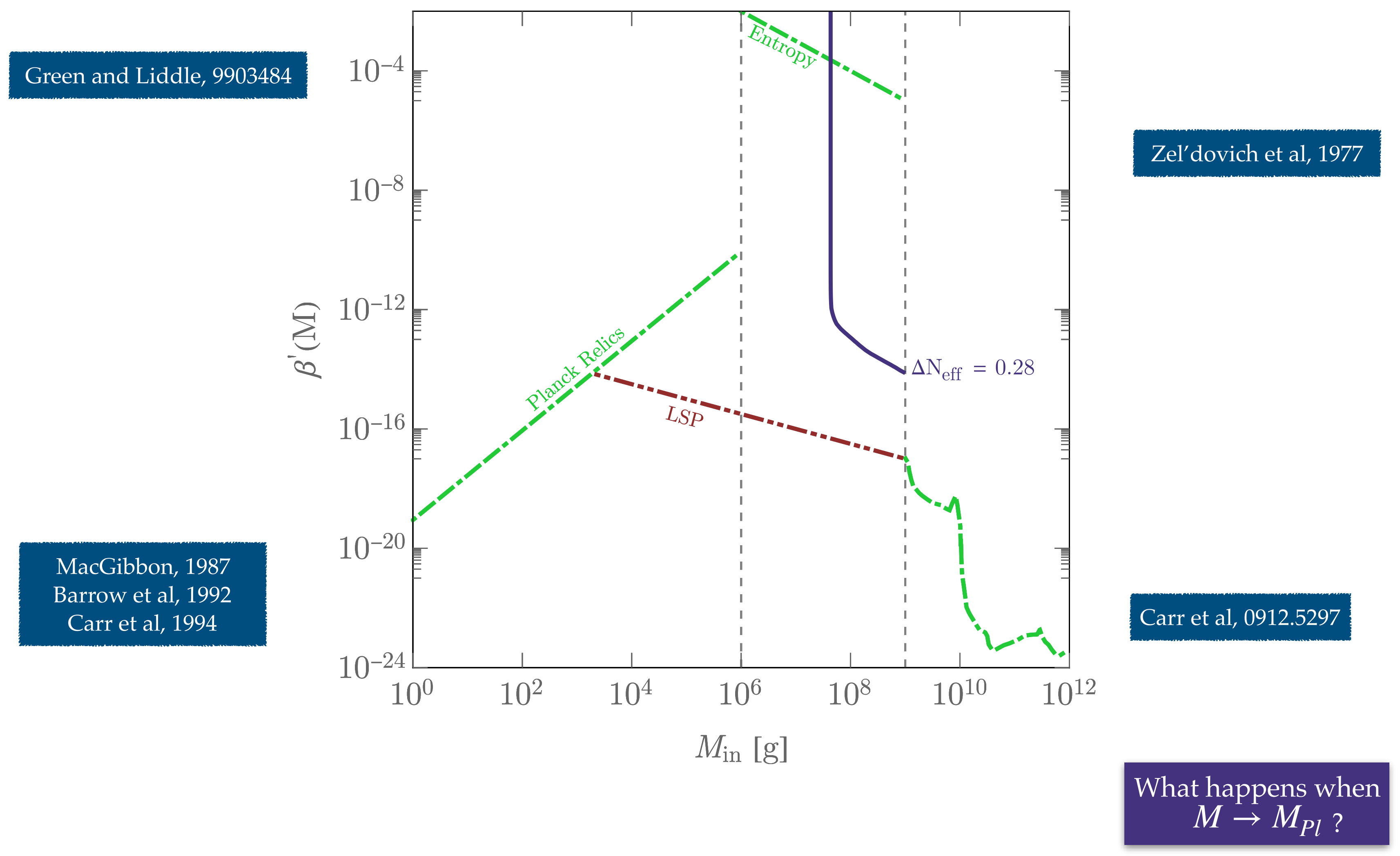




\section{Constraints in the Dirac neutrino case}

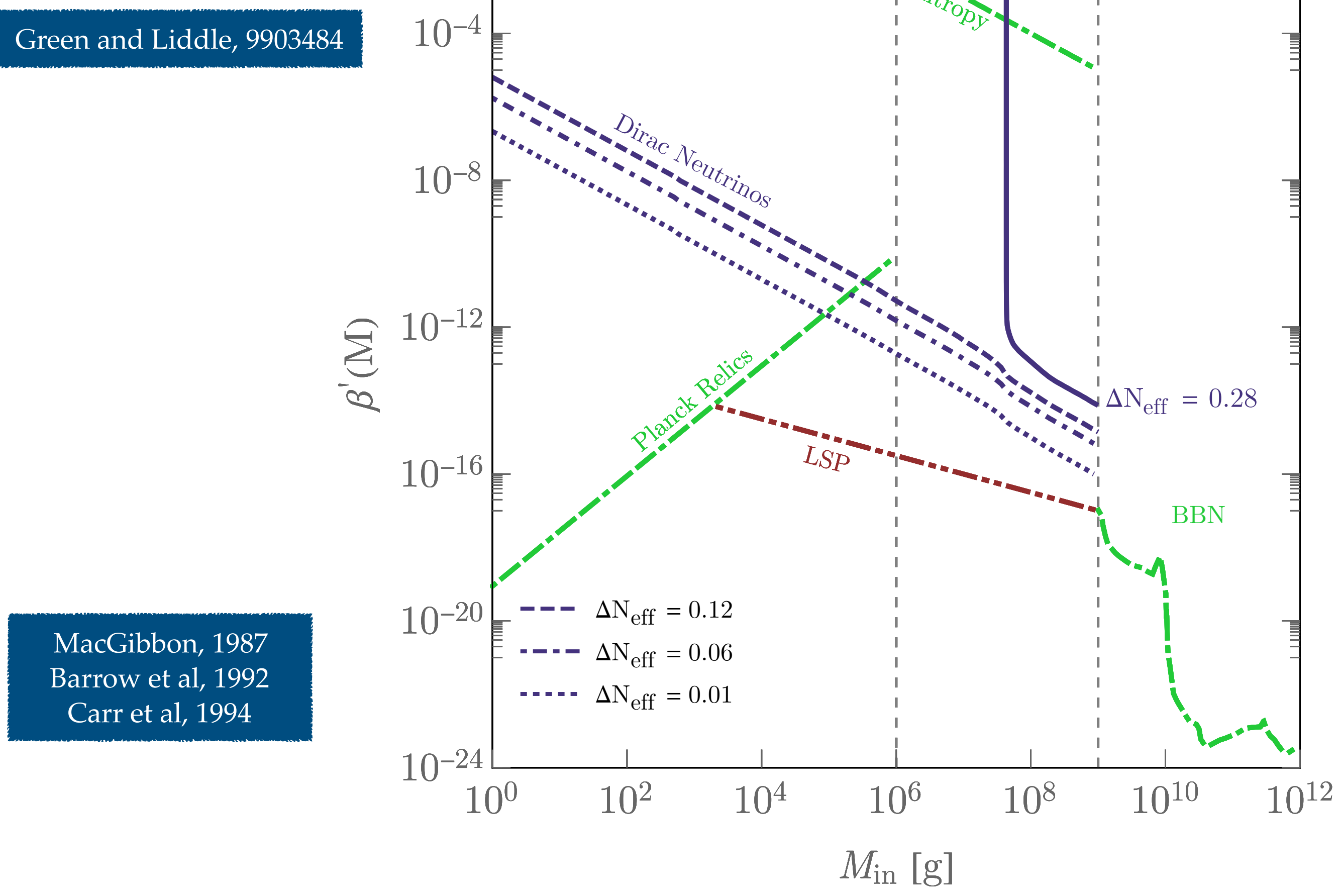

Zel'dovich et al, 1977

Carr et al, 0912.5297

What happens when $M \rightarrow M_{P l}$ ? 


\section{Constraints in the Dirac neutrino case}

Green and Liddle, 9903484

MacGibbon, 1987 Barrow et al, 1992 Carr et al, 1994

Tension between the early and late measurements of the Hubble constant

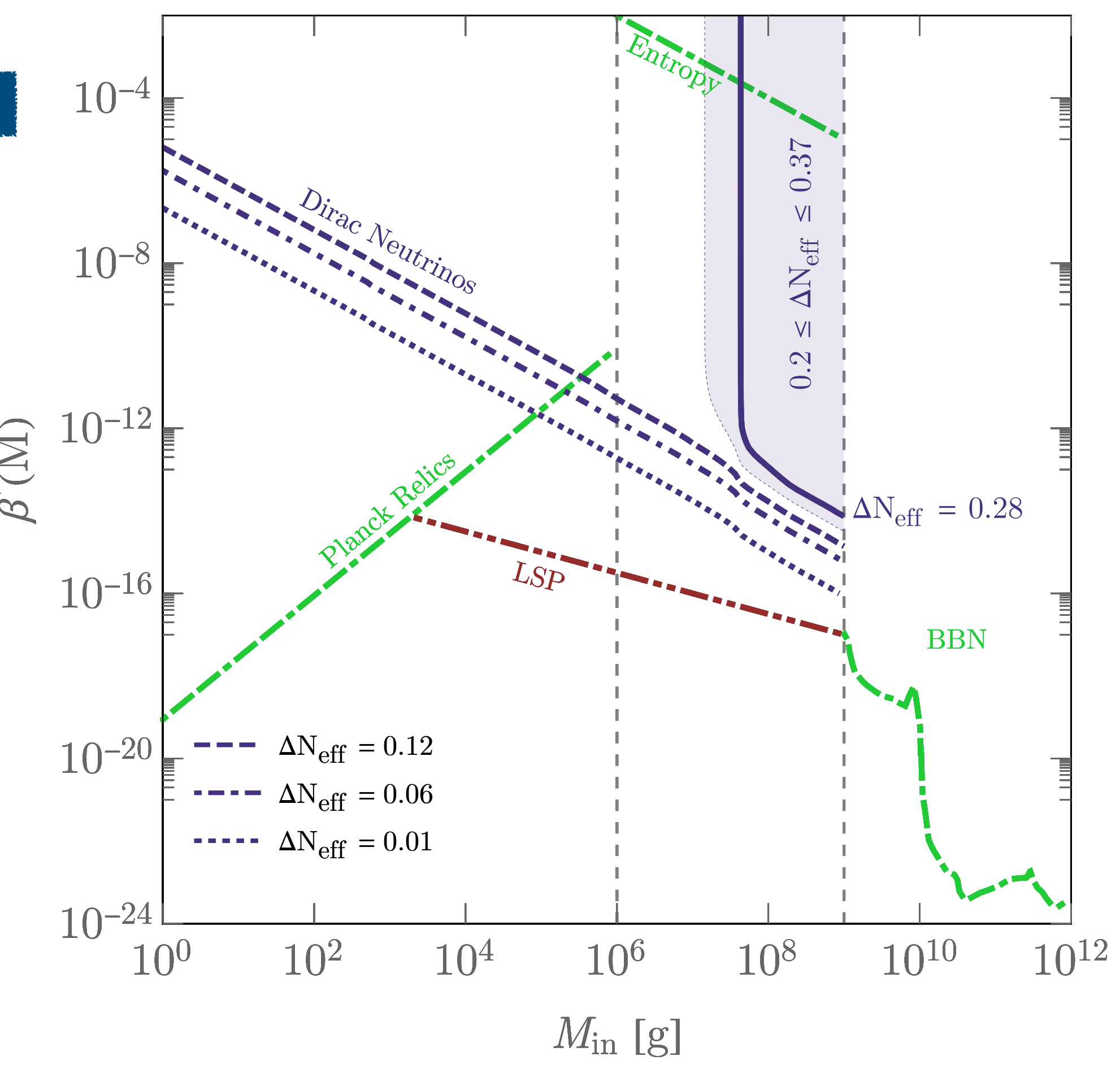

What happens when $M \rightarrow M_{P l}$ ? 


\section{Constraints in the Dirac neutrino case}

Let us consider the

minimal extension

$$
\mathscr{L}_{Y}=-Y_{\nu}^{a b} \overline{L_{L}^{a}} \widetilde{H} \nu_{b R}
$$

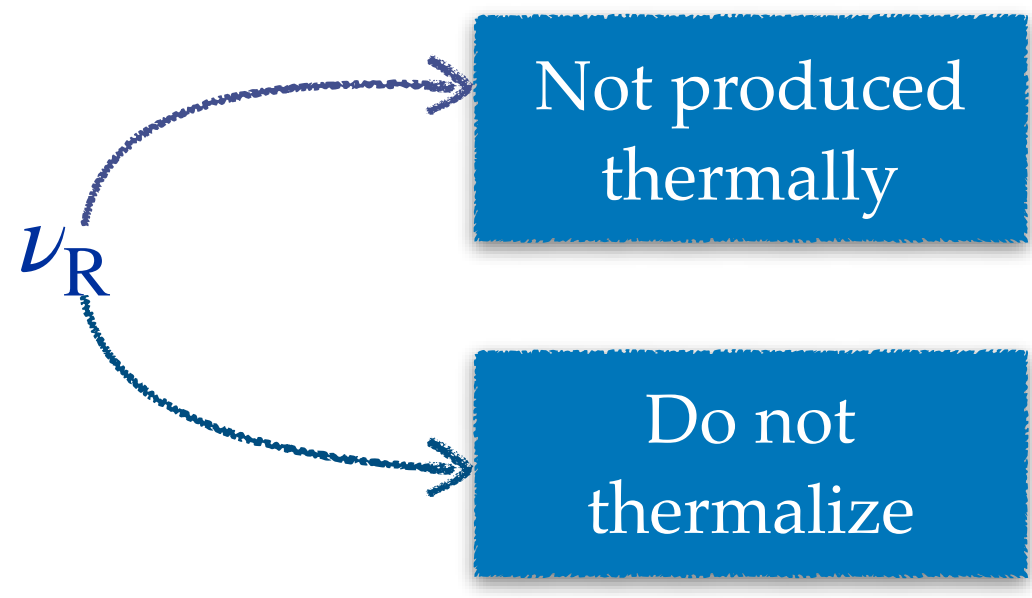

Antonelli et al, 1981

Chen et al, 1509.00481

$$
\begin{aligned}
& \text { Friedmann- } \\
& t_{i}, T_{\mathrm{f}} \\
& t_{\mathrm{EV}}, T_{\mathrm{EV}} \\
& \Delta N_{\mathrm{eff}}=\left\{\frac{8}{7}\left(\frac{4}{11}\right)^{-\frac{4}{3}}+N_{\mathrm{eff}}^{\mathrm{SM}}\right\} \frac{\rho_{\nu_{\mathrm{R}}}\left(T_{\mathrm{EV}}\right)}{\rho_{\mathrm{R}}\left(T_{\mathrm{EV}}\right)}\left(\frac{g_{*}\left(T_{\mathrm{EV}}\right)}{g_{*}\left(T_{\mathrm{EQ}}\right)}\right)\left(\frac{g_{* S}\left(T_{\mathrm{EQ}}\right)}{g_{* S}\left(T_{\mathrm{EV}}\right)}\right)^{\frac{4}{3}}
\end{aligned}
$$

\section{Matter-}




\section{Constraints in the Dirac neutrino case}

Let us consider the

minimal extension

$$
\mathscr{L}_{Y}=-Y_{\nu}^{a b} \overline{L_{L}^{a}} \widetilde{H} \nu_{b R}
$$

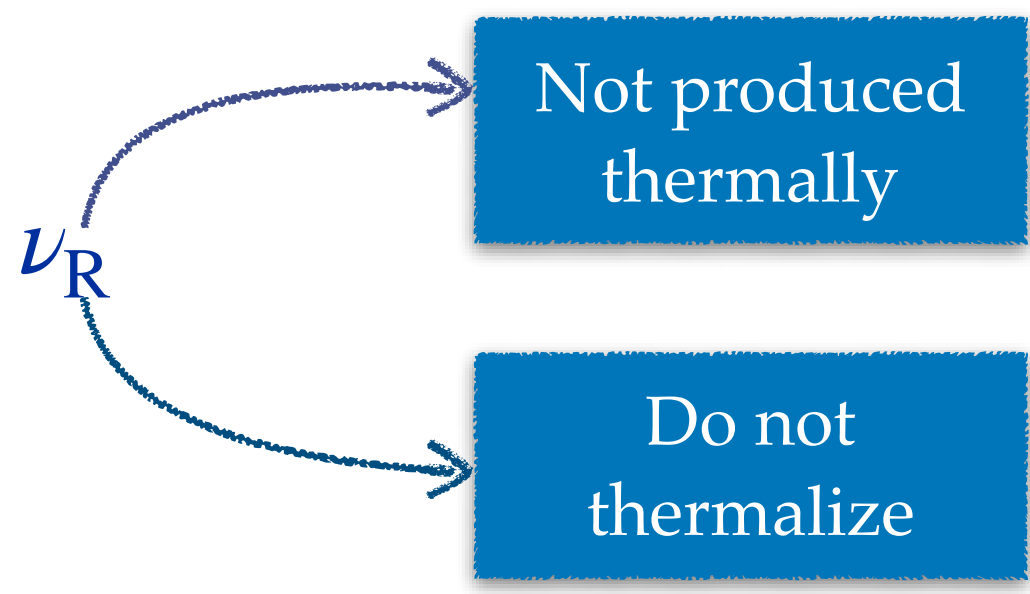

Antonelli et al, 1981

Chen et al, 1509.00481

\section{Friedmann- \\ Boltzmann \\ Equations}

PBHs are formed

Non-standard cosmology?

$$
t_{i}, T_{\mathrm{f}}
$$

$$
t_{\mathrm{EV}}, T_{\mathrm{EV}}
$$$$
t_{\mathrm{EQ}}, T_{\mathrm{EQ}}
$$

$t_{0}$

$$
\Delta N_{\mathrm{eff}}=\left\{\frac{8}{7}\left(\frac{4}{11}\right)^{-\frac{4}{3}}+N_{\mathrm{eff}}^{\mathrm{SM}}\right\} \frac{\rho_{\nu_{\mathrm{R}}}\left(T_{\mathrm{EV}}\right)}{\rho_{\mathrm{R}}\left(T_{\mathrm{EV}}\right)}\left(\frac{g_{*}\left(T_{\mathrm{EV}}\right)}{g_{*}\left(T_{\mathrm{EQ}}\right)}\right)\left(\frac{g_{* S}\left(T_{\mathrm{EQ}}\right)}{g_{* S}\left(T_{\mathrm{EV}}\right)}\right)^{\frac{4}{3}}
$$




\section{Diffuse neutrino flux from $\mathrm{PBHs}$}

Could we measure

these neutrinos?

$$
\frac{d \Phi_{\mathrm{PBH}}^{\nu}}{d p_{0}}=\int_{t_{i}}^{\min \left(t_{0}, \tau\right)} d t \frac{d \Omega}{4 \pi} \frac{a_{0}}{a_{t}}\left(\frac{a_{i}}{a_{0}}\right)^{3} \frac{\rho_{\mathrm{PBH}}^{i}}{M_{i}} \frac{d^{2} N_{\nu}}{d p d t}\left(M(t), p_{0} a_{0} / a_{t}\right)
$$




\section{Diffuse neutrino flux from $\mathrm{PBHs}$}

Could we measure

these neutrinos?

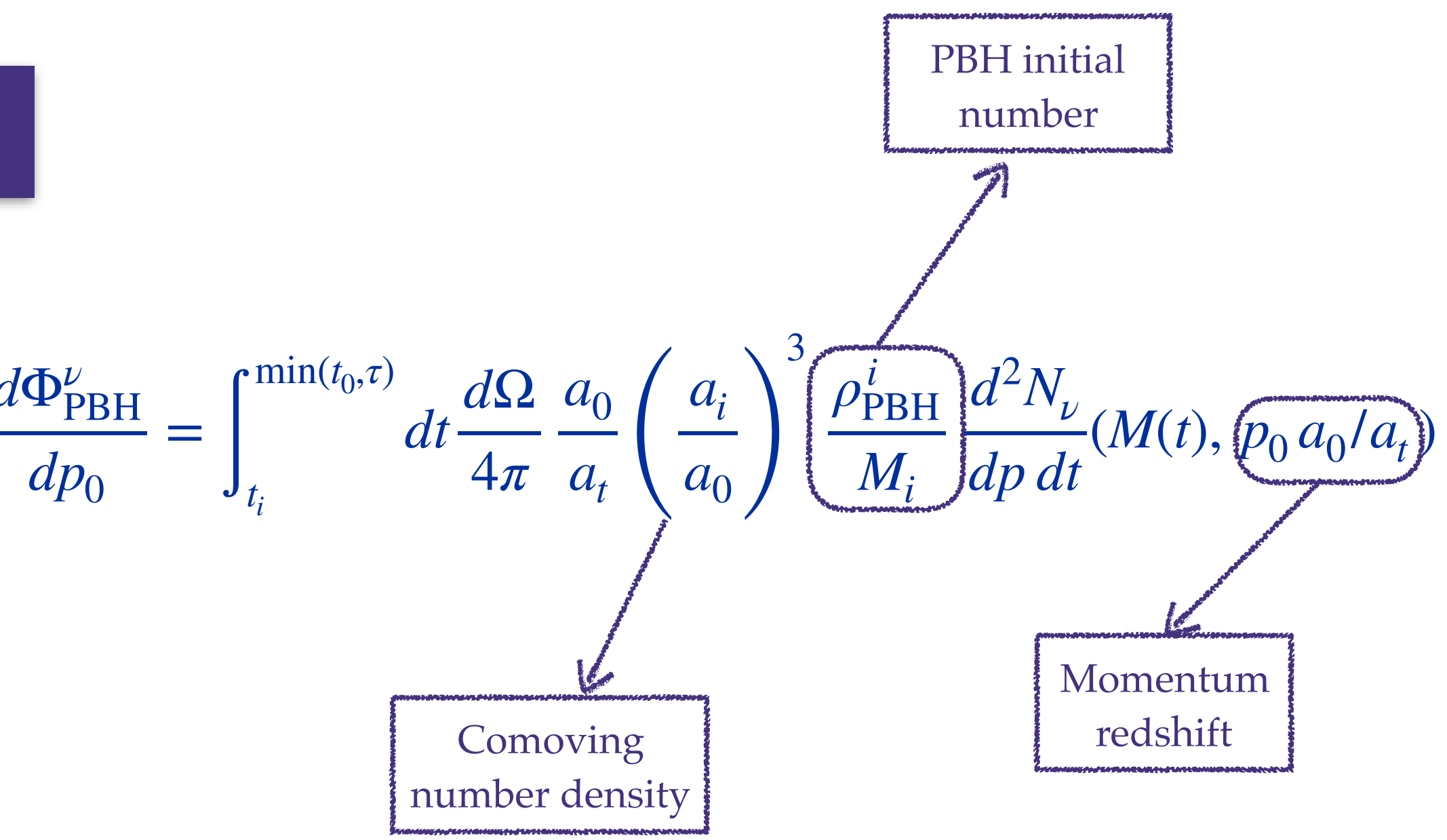




\section{Diffuse neutrino flux from PBHs}

Could we measure

these neutrinos?

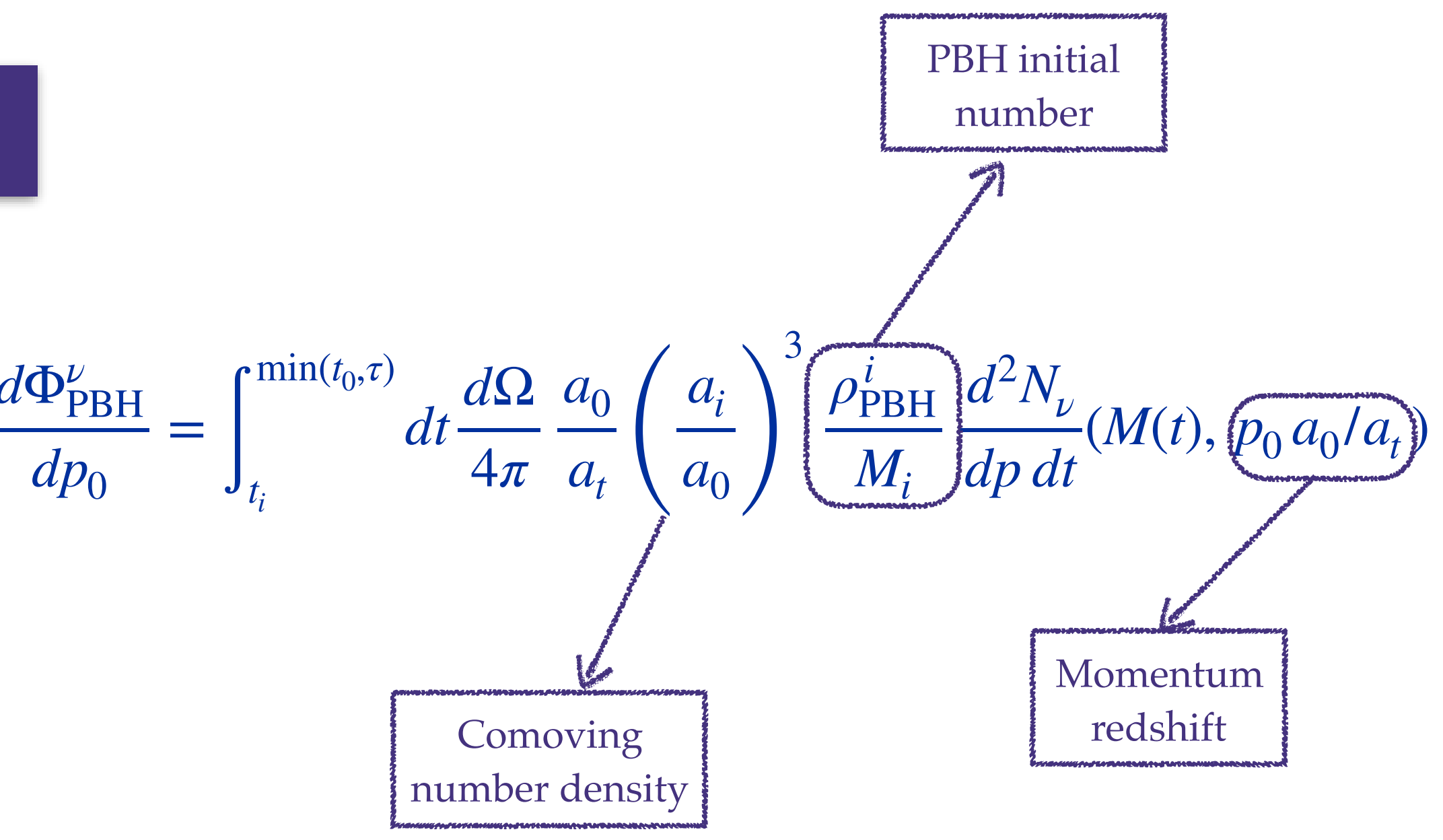

$$
\begin{gathered}
\frac{a_{i}}{a_{0}}=\left(\frac{a_{i}}{a_{\mathrm{EV}}}\right)\left(\frac{g_{* S}\left(T_{\mathrm{EV}}\right)}{g_{* S}\left(T_{\mathrm{EQ}}\right)}\right)^{\frac{1}{3}}\left(\frac{T_{\mathrm{EV}}}{T_{\mathrm{EQ}}}\right)\left(1+z_{\mathrm{EQ}}\right) \\
\begin{array}{c}
\text { Matter-radiation } \\
\text { redshift }
\end{array}
\end{gathered}
$$




\section{Diffuse flux}
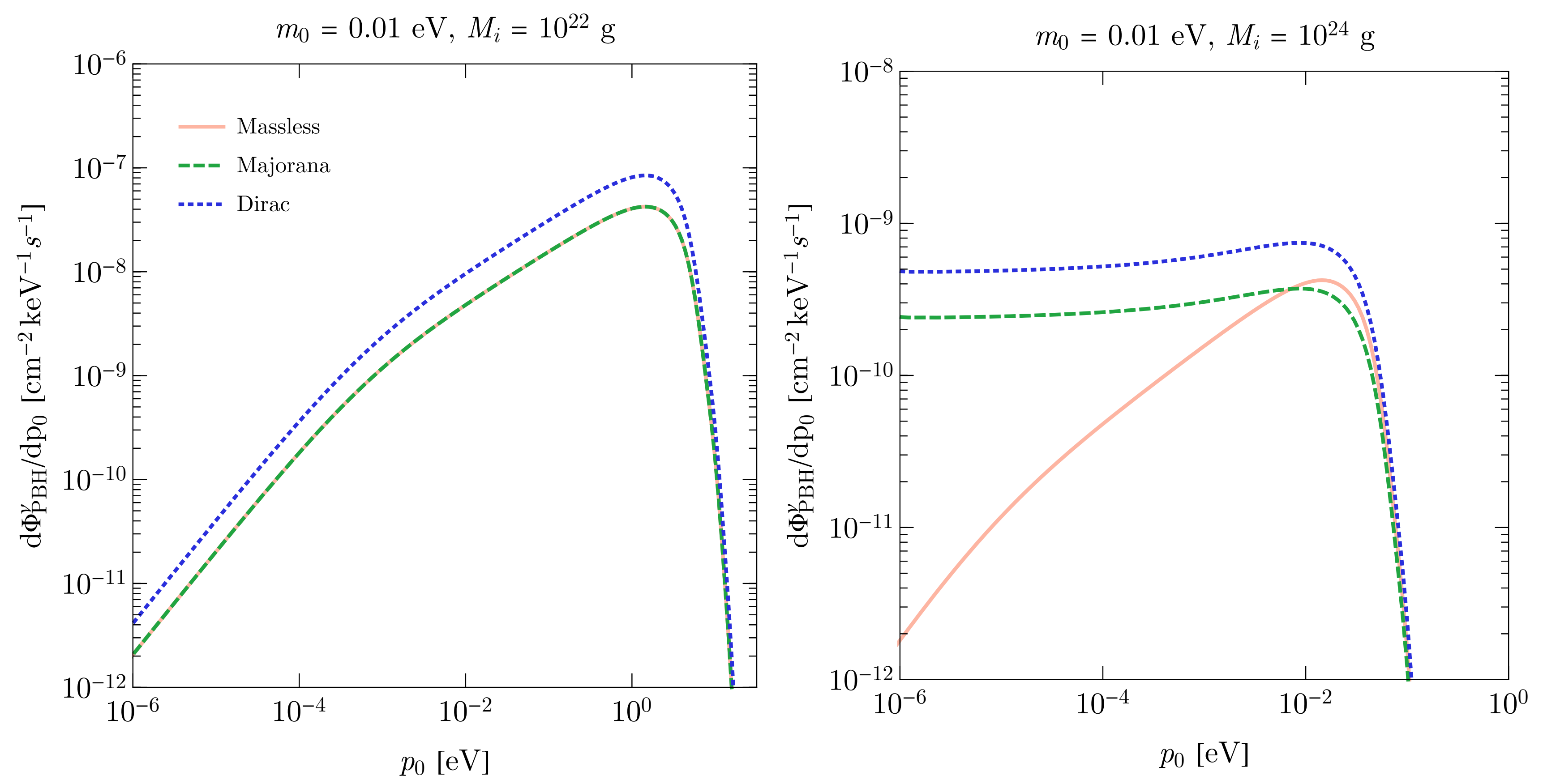


\section{Diffuse flux}
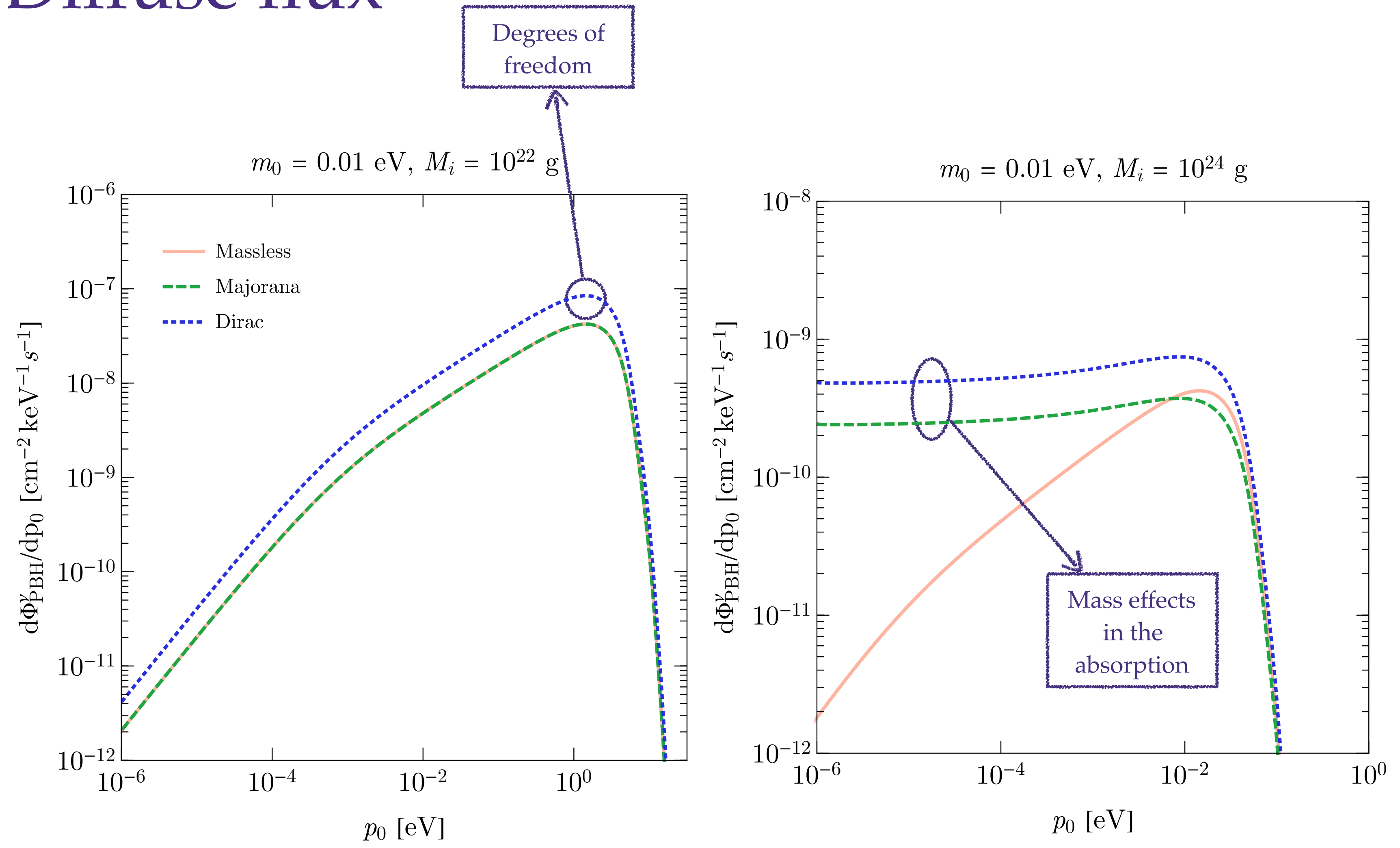


\section{Diffuse flux}

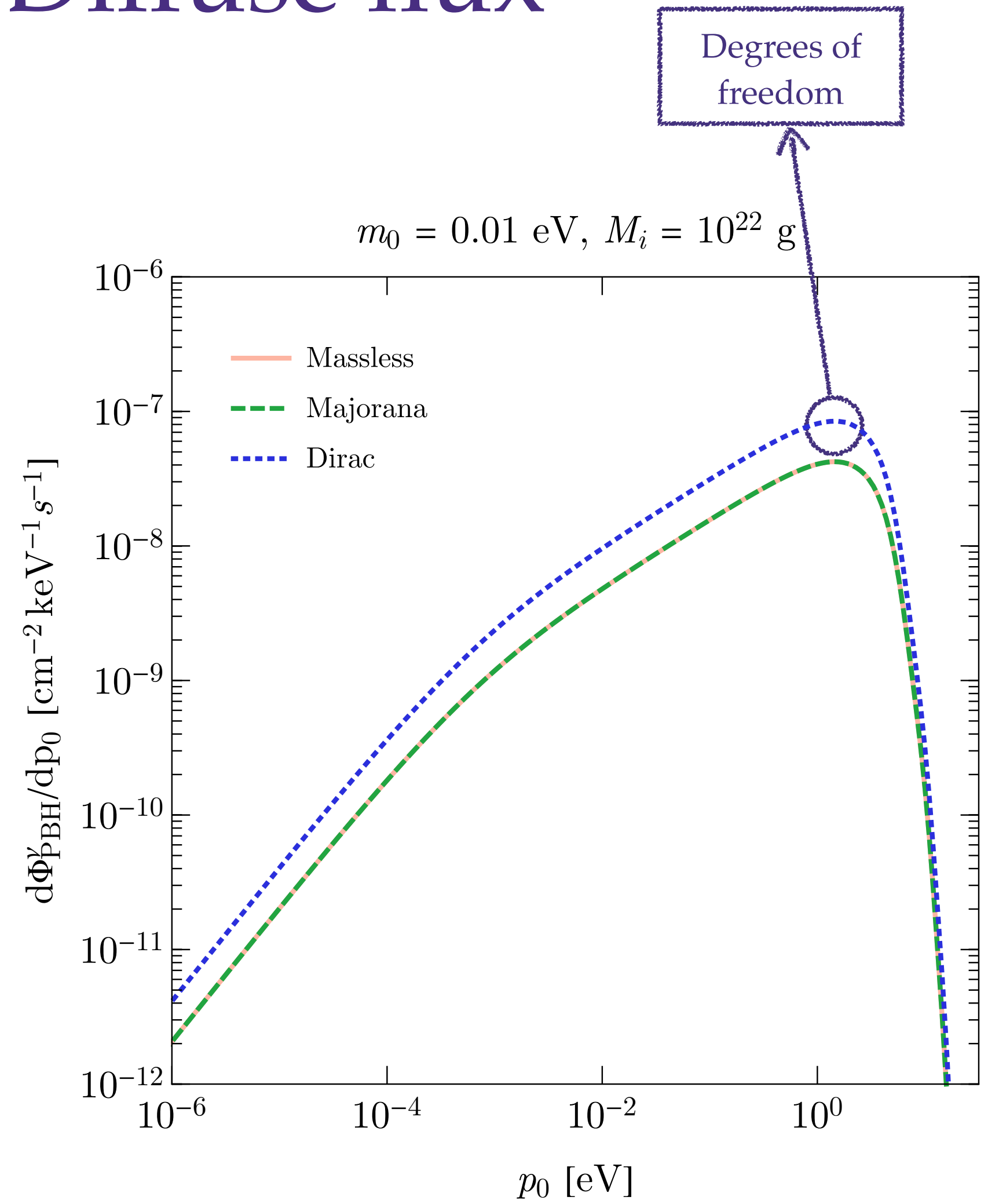

Information on the neutrino mass and nature

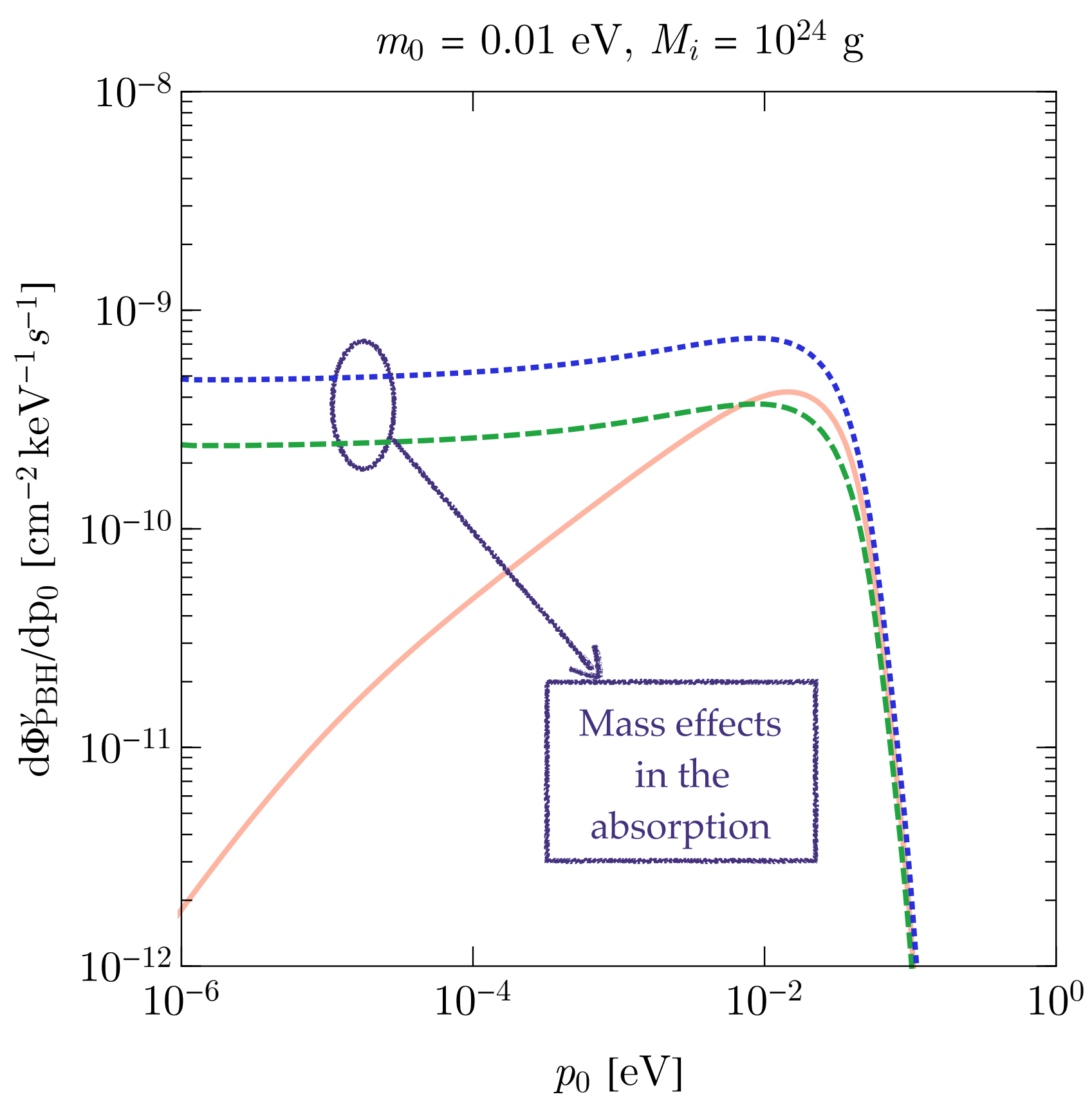

Could it be detectable? 


\section{Diffuse flux from non-evaporating $\mathrm{PBH}$}

Total Majorana $v$ Flux, $m_{0}=0.01 \mathrm{eV}, M_{i}>M_{*}$

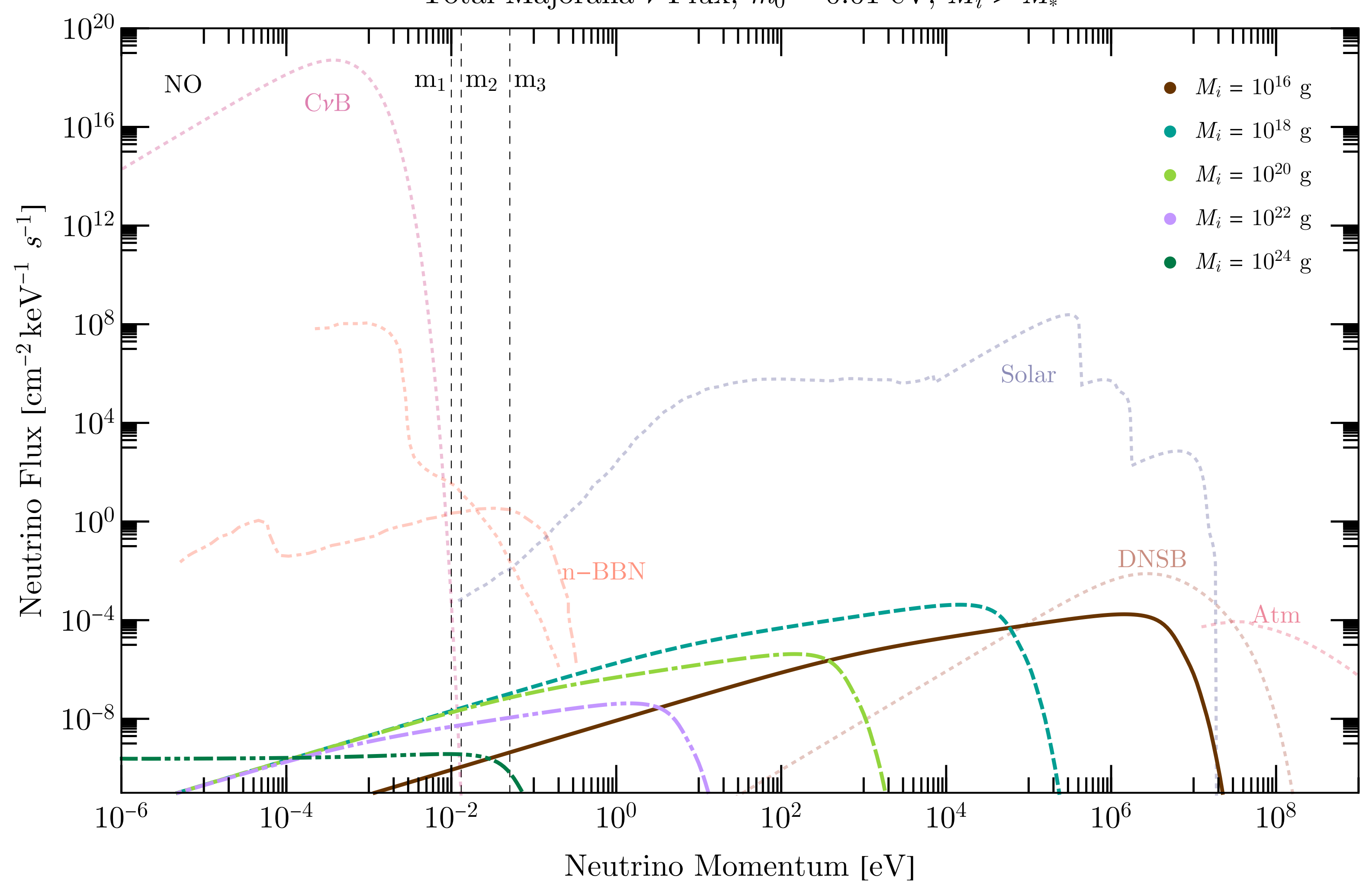




\section{Diffuse flux from non-evaporating $\mathrm{PBHs}$}

Total Majorana $v$ Flux, $m_{0}=0.01 \mathrm{eV}, M_{i}>M_{*}$

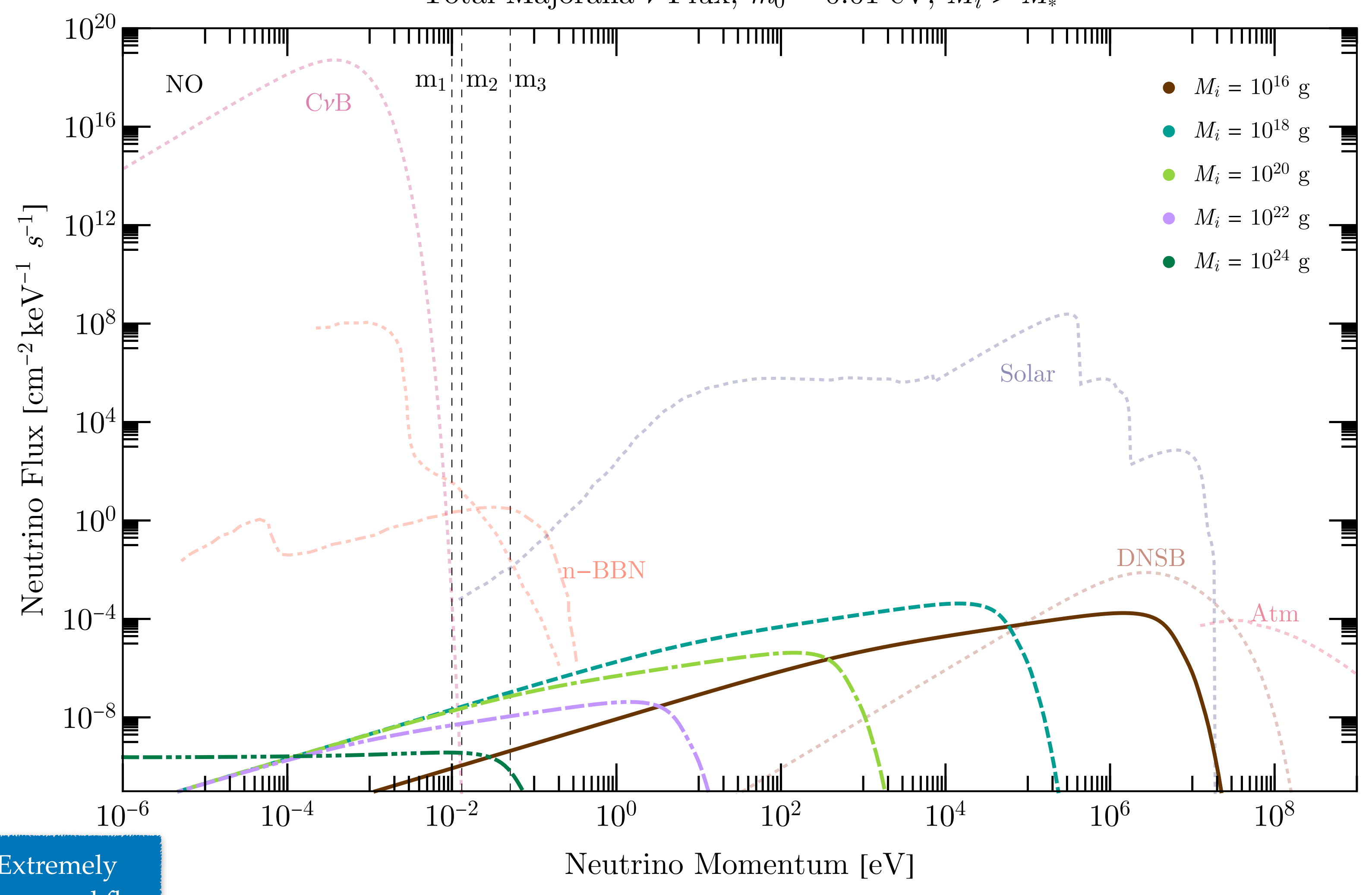




\section{Diffuse flux of $\mathrm{RH}$ neutrinos from $\mathrm{PBH}$}

Total RH Neutrino Flux, $m_{0}=0.01 \mathrm{eV}, M_{i} \leq M_{*}$

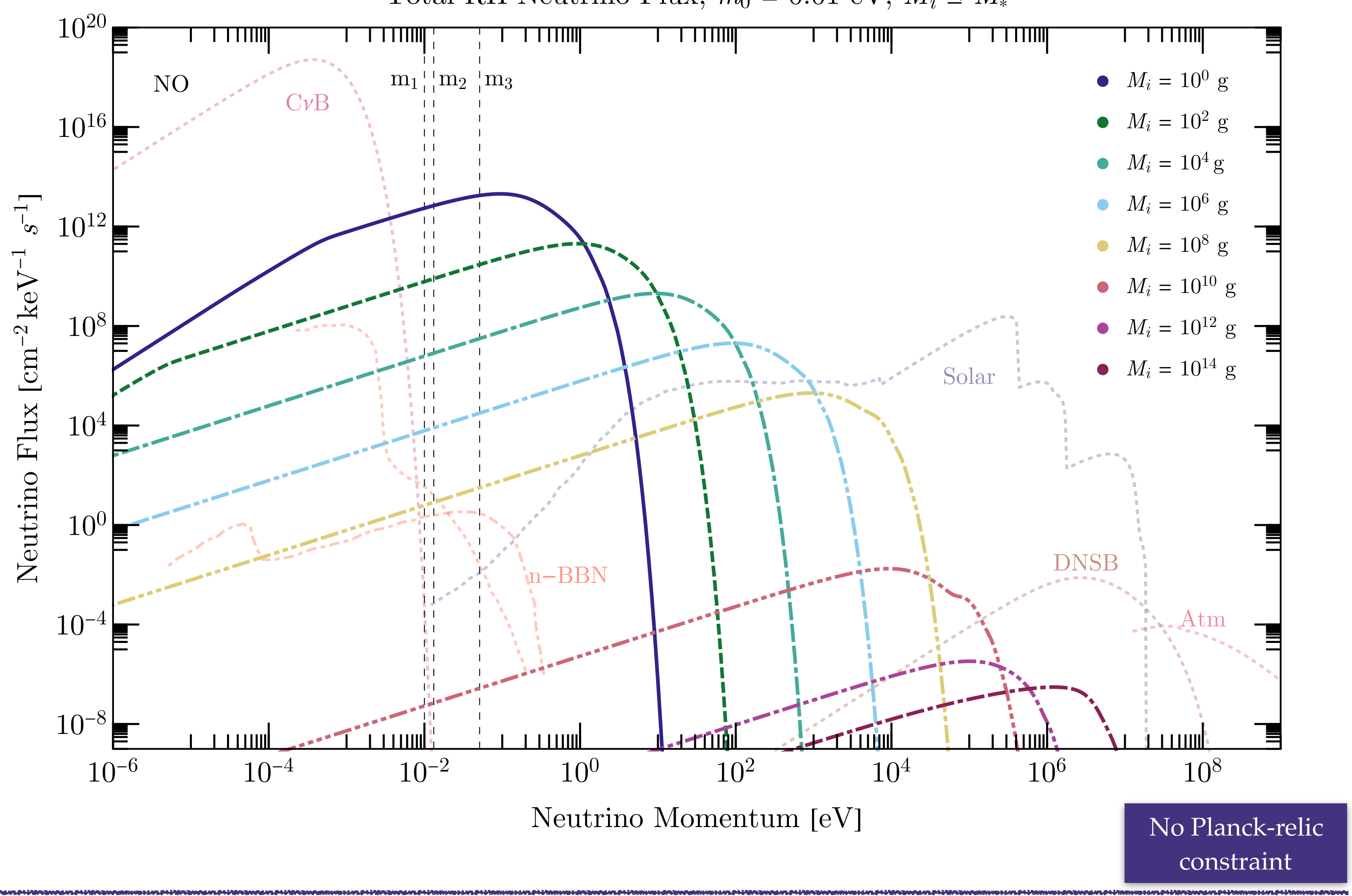




\section{Diffuse flux of $\mathrm{RH}$ neutrinos from $\mathrm{PBH}$}

Total RH Neutrino Flux, $m_{0}=0.01 \mathrm{eV}, M_{i} \leq M_{*}$

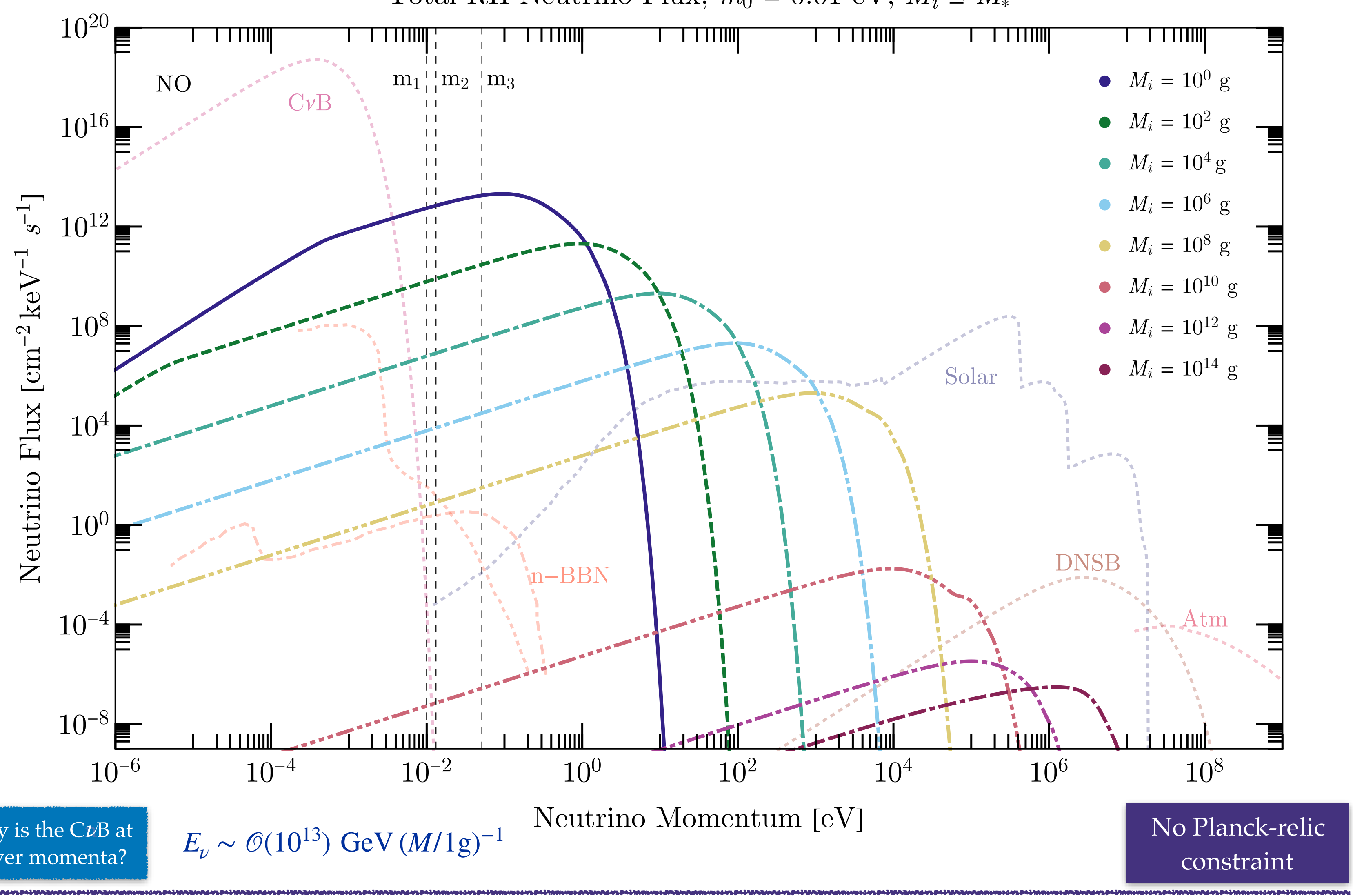




\section{Detection?}




\section{Detection?}

\section{Helicity}

suppression

$m_{0}=0.01 \mathrm{eV}$

$$
\frac{m_{\nu}}{E_{\nu}} \sim 10^{-1} \longrightarrow M=1 \mathrm{~g}
$$




\section{Detection?}

\section{Helicity}

suppression

$m_{0}=0.01 \mathrm{eV}$

$$
\frac{m_{\nu}}{E_{\nu}} \sim 10^{-1} \longrightarrow M=1 \mathrm{~g}
$$

Akhmedov's talk

PTOLEMY?

$$
\nu_{a}+n \rightarrow p^{+}+e^{-}
$$

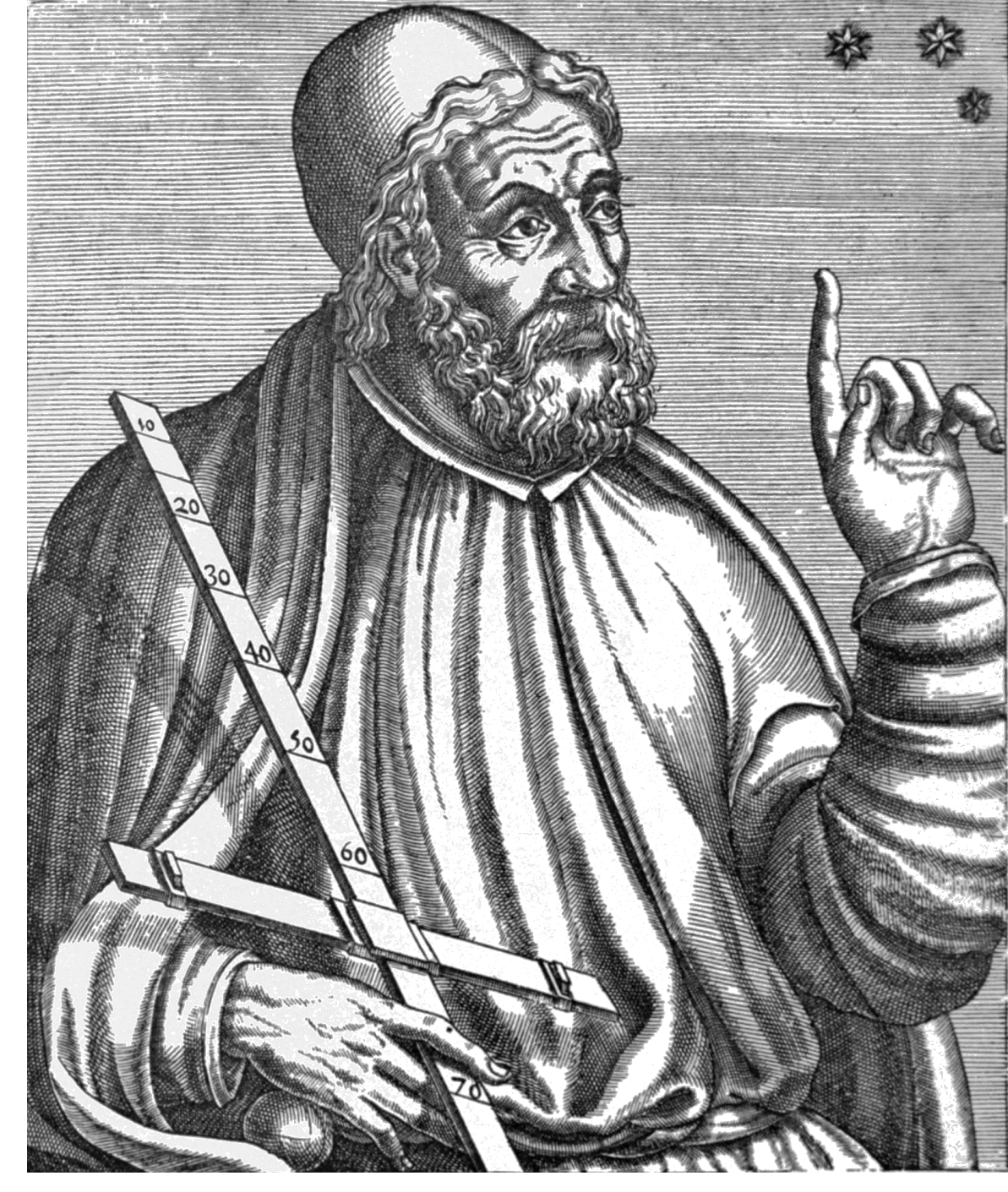

$\Gamma_{\mathrm{C} \nu \mathrm{B}}^{\mathrm{D}} \sim 40[\mathrm{~kg}-\mathrm{year}]^{-1}$ 


\section{Detection?}

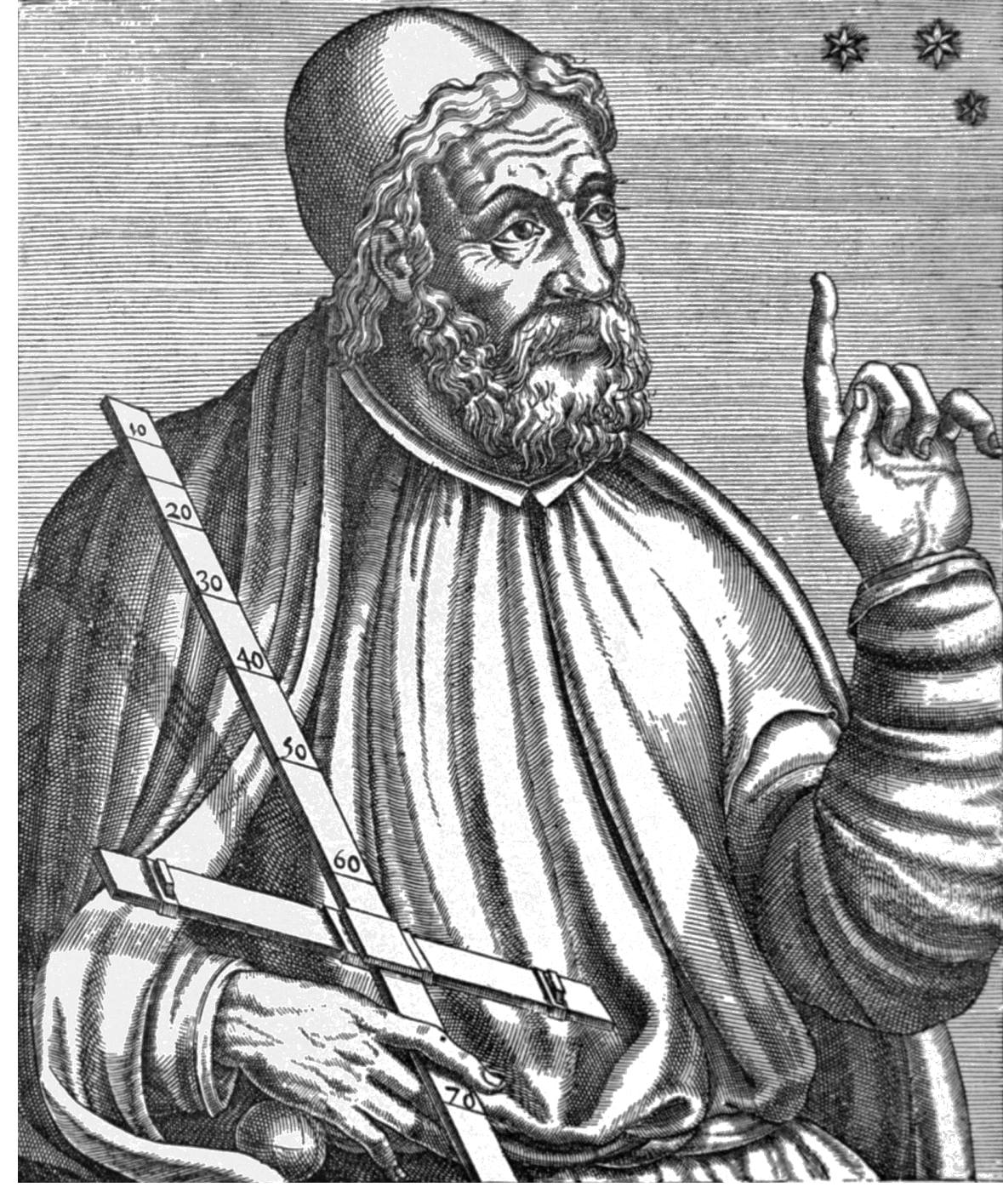

Akhmedov's talk

PTOLEMY?

$\nu_{a}+n \rightarrow p^{+}+e^{-}$

$\Gamma_{\mathrm{C} \nu \mathrm{B}}^{\mathrm{D}} \sim 40[\mathrm{~kg}-\mathrm{year}]^{-1}$

$\Gamma_{\mathrm{PBH}}^{\nu} \sim 10^{-2}[\mathrm{~kg}-\text { year }]^{-1}$

PBH RH

flux is still

suppressed
Are there other possible ways to try to detect this $\mathrm{RH}$ neutrino flux? 


\section{Conclusions}

- BHs are sources of neutrinos in mass eigenstates

- The PBH evaporation depends on whether neutrinos are Dirac or Majorana particles

- In the Dirac scenario, there is not a helicity suppression of the emission of righthanded neutrinos

- We derived a constraint on the initial PBH fraction given the measurement of Neff by Planck

- For certain values, it is possible to ease the Hubble measurement tension

- The diffuse flux of RH neutrinos can be large, but more careful analysis on its possible detection should be performed 


\section{Thank you!}

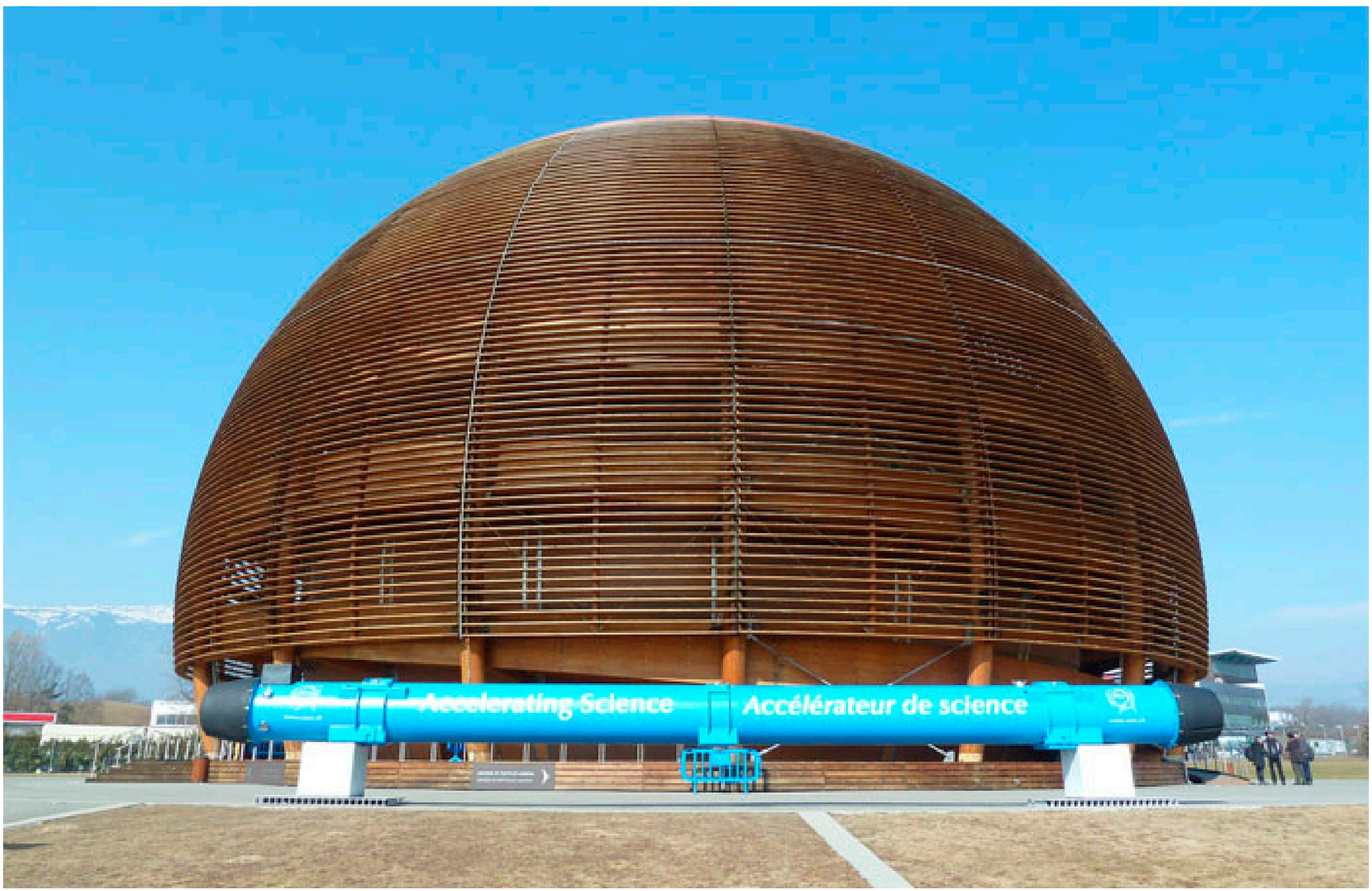




\section{Backup slides}




\section{Evaporation function}

$$
\begin{aligned}
& \varepsilon_{N}(M)= 2 f_{1}+4 f_{1 / 2}^{1}\left\{\sum_{\ell=e, \mu, \tau} \exp \left[-\frac{M}{\beta_{1 / 2} M_{\ell}}\right]+3 \sum_{q} \exp \left[-\frac{M}{\beta_{1 / 2} M_{q}}\right]\right\} \\
&+2 \eta_{\nu}^{N} f_{1 / 2}^{0} \sum_{a=1,2,3} \exp \left[-\frac{M}{\beta_{1 / 2} M_{a}}\right] \\
&+16 f_{1} \exp \left[-\frac{M}{\beta_{1} M_{g}}\right] \\
&+3 f_{1}\left\{2 \exp \left[-\frac{M}{\beta_{1} M_{W}}\right]+\exp \left[-\frac{M}{\beta_{1} M_{Z}}\right]\right\}+f_{0} \exp \left[-\frac{M}{\beta_{0} M_{H}}\right] \\
& \beta_{s}=\left\{\begin{array}{ll}
2.66 \text { for } s=0 \\
4.53 \text { for } s=
\end{array} \quad \frac{1}{2} \quad f_{s}=\left\{\begin{array}{ll}
0.267 & \text { for } s=0 \\
0.060 & \text { for } s=1 \\
0.007 & \text { for } s=2
\end{array} \quad f_{1 / 2}^{q}= \begin{cases}0.147 & \text { for } q=0 \text { (neutral) } \\
0.142 & \text { for } q=1 \text { (charged) }\end{cases} \right.\right.
\end{aligned}
$$




\section{Absorption cross section}

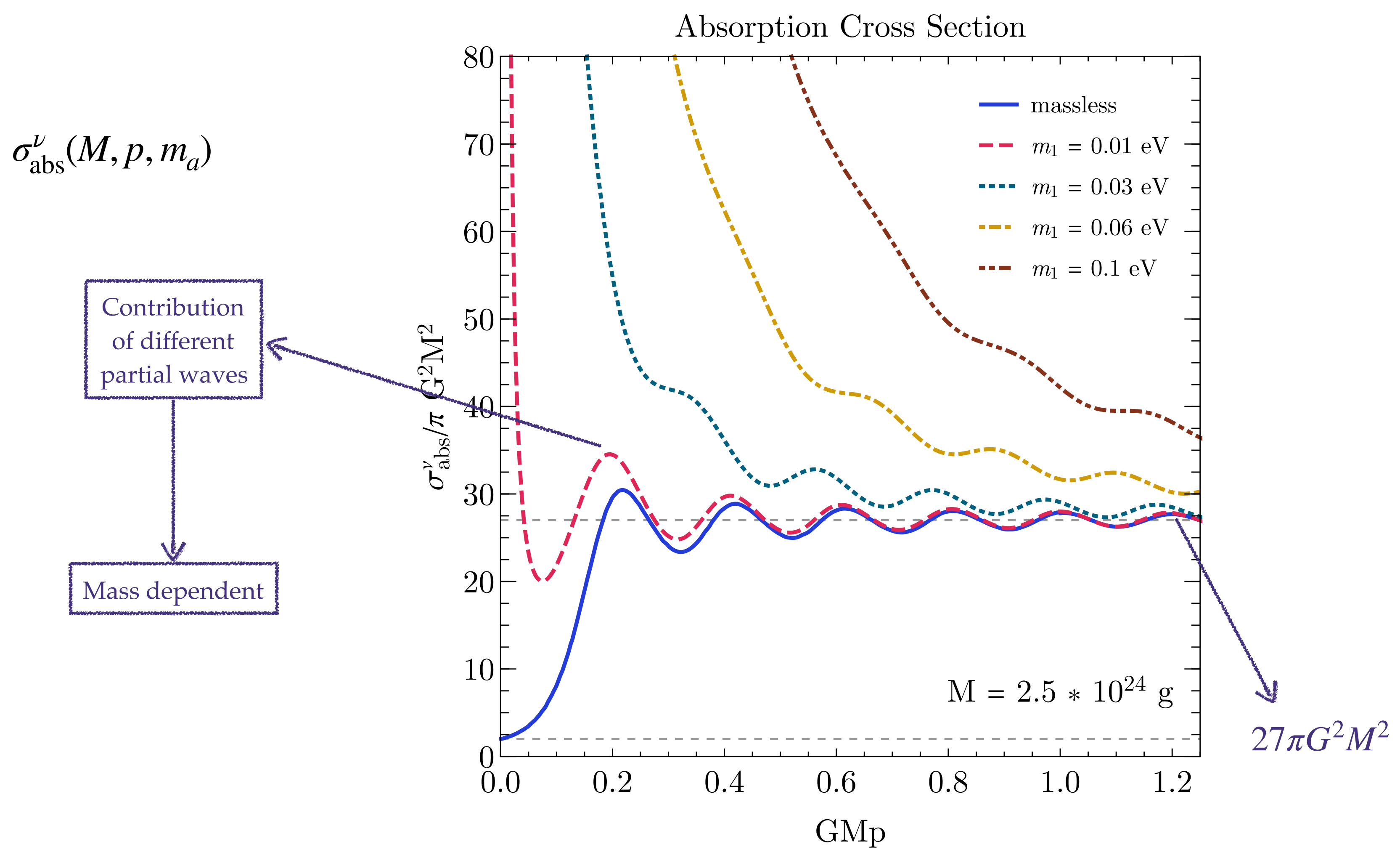




\section{Constraints in the Dirac neutrino case}

Let us consider the

minimal extension

$$
\mathscr{L}_{Y}=-Y_{\nu}^{a b} \overline{L_{L}^{a}} \widetilde{H} \nu_{b R}
$$

\section{Do not thermalize}

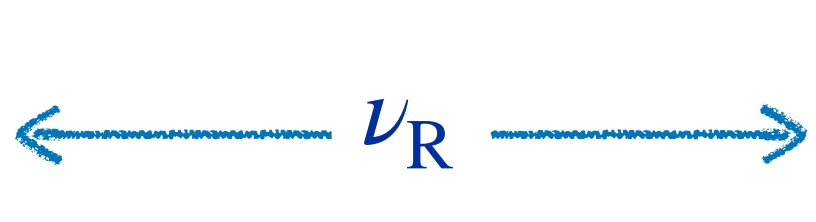

\section{Not produced}

thermally

\section{Assuming an initial}

radiation-dominated

Universe

\section{Formation Temperature}

\section{Initial fraction}

$$
T_{f}=\left(\frac{45}{16 \pi^{3} G^{3}}\right)^{\frac{1}{4}} g_{*}\left(T_{f}\right)^{-\frac{1}{4}} \gamma^{\frac{1}{2}} M_{i}^{-\frac{1}{2}}
$$




\section{Constraints in the Dirac neutrino case}

Friedmann and

Boltzmann Equations

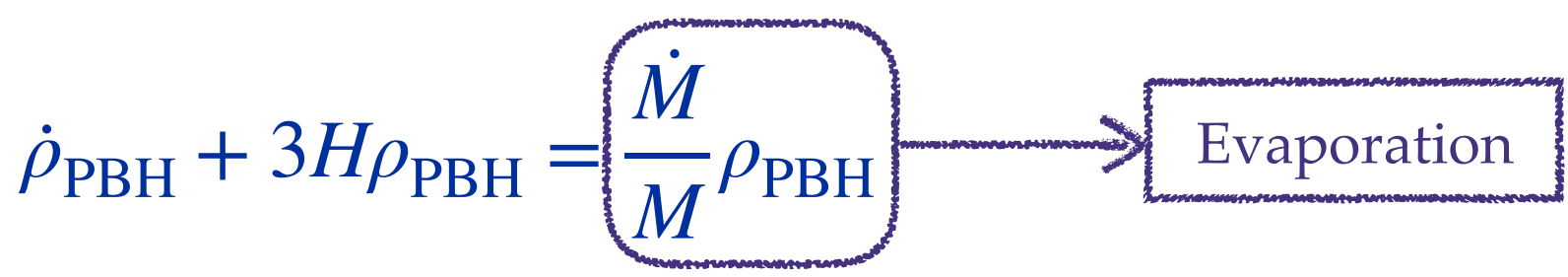

\section{BR into SM}

$$
\dot{\rho}_{\mathrm{R}}+4 H \rho_{\mathrm{R}}=-\frac{\varepsilon_{\mathrm{SM}}(M)}{\varepsilon_{\mathrm{D}}(M)} \frac{\dot{M}}{M} \rho_{\mathrm{PBH}}
$$

$$
\dot{\rho}_{\nu_{\mathrm{R}}}+4 H \rho_{\nu_{\mathrm{R}}}=-\frac{\varepsilon_{\nu_{\mathrm{R}}}}{\varepsilon_{\mathrm{D}}(M)} \frac{\dot{M}}{M} \rho_{\mathrm{PBH}}
$$$$
\varepsilon_{\nu_{\mathrm{R}}}=6 * 0.147
$$

$$
H^{2}=\frac{8 \pi G}{3}\left(\rho_{\mathrm{PBH}}+\rho_{\mathrm{R}}+\rho_{\nu_{\mathrm{R}}}\right)
$$

$$
\frac{\dot{T}}{T}=-\frac{H}{\Delta}-\frac{1}{\Delta} \frac{\dot{M}}{M} \frac{g_{*}(T)}{g_{* S}(T)} \frac{\rho_{\mathrm{PBH}}}{4\left(\rho_{\mathrm{R}}+\rho_{\nu_{\mathrm{R}}}\right)}
$$

$\Delta=1+\frac{T}{3 g_{* S}(T)} \frac{d g_{* S}(T)}{d T}$

$$
\beta^{\prime} \gtrsim 2.5 \times 10^{-14}\left(\frac{g_{*}\left(T_{f}\right)}{106.75}\right)^{-\frac{1}{4}}\left(\frac{M_{i}}{10^{8} \mathrm{~g}}\right)^{-1}\left(\frac{\varepsilon_{D}\left(M_{i}\right)}{15.35}\right)^{\frac{1}{2}}
$$




\section{Constraints in the Dirac neutrino case}

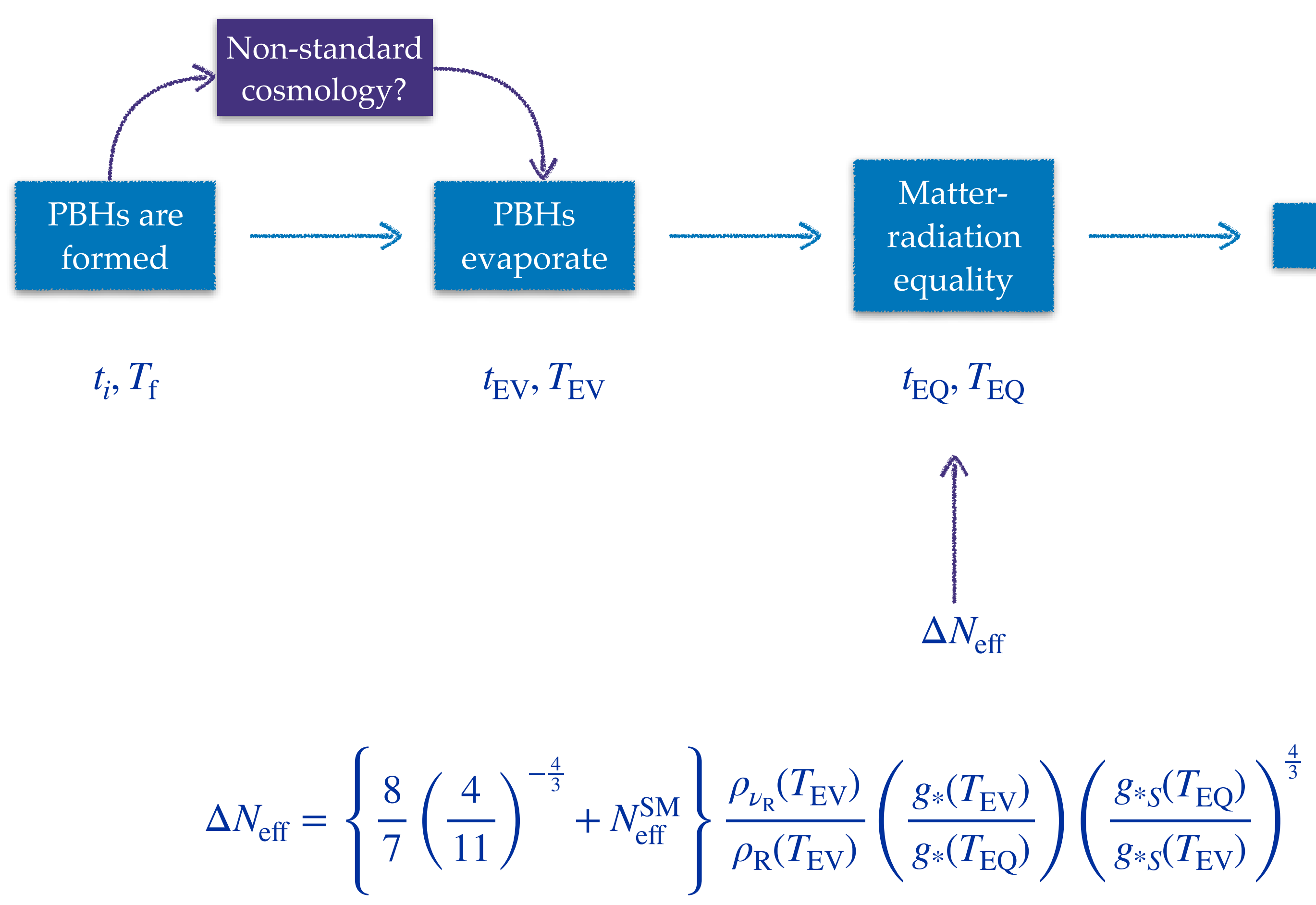

Hooper et al, 1905.01301 


\section{Neutrino spectrum}

\section{Hawking spectrum}

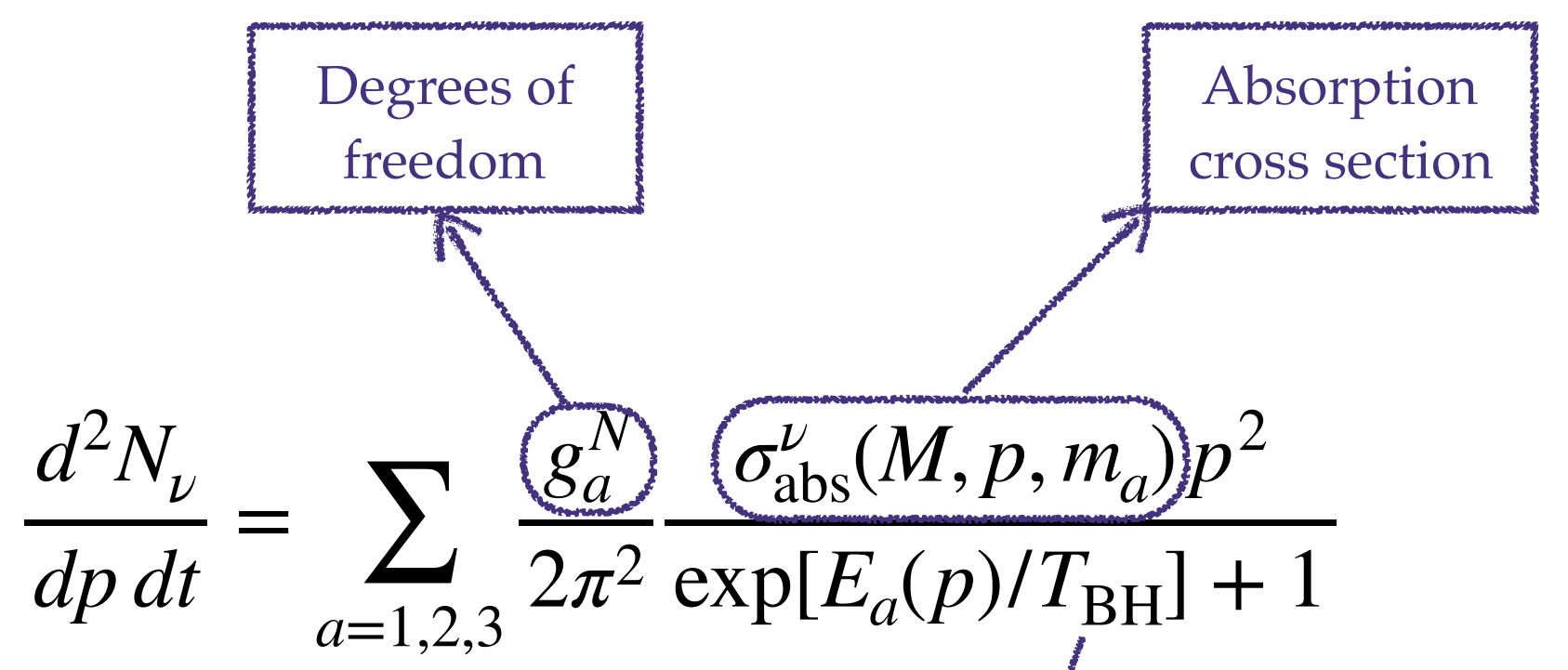

$$
\alpha_{g}^{a}=G M m_{a}
$$

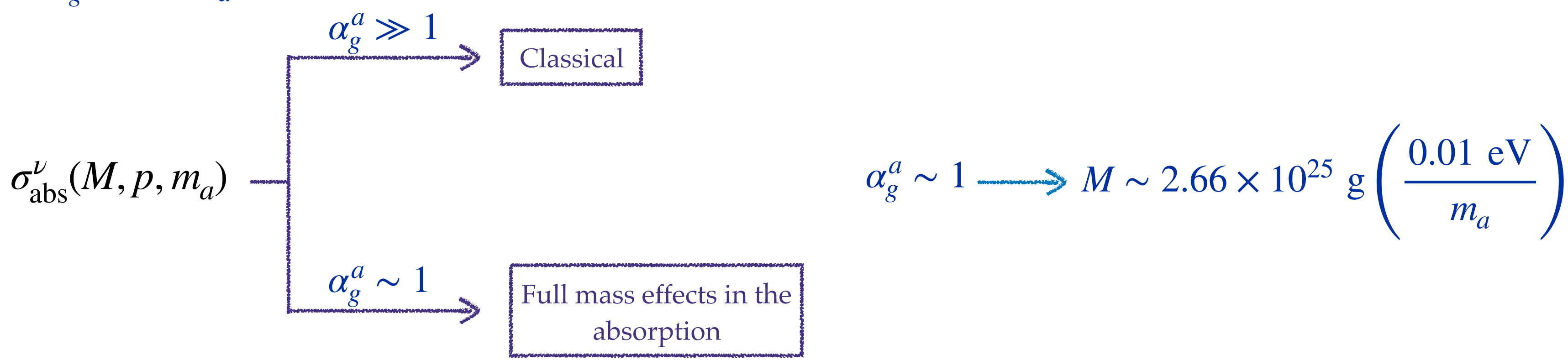




\section{Hawking spectrum}
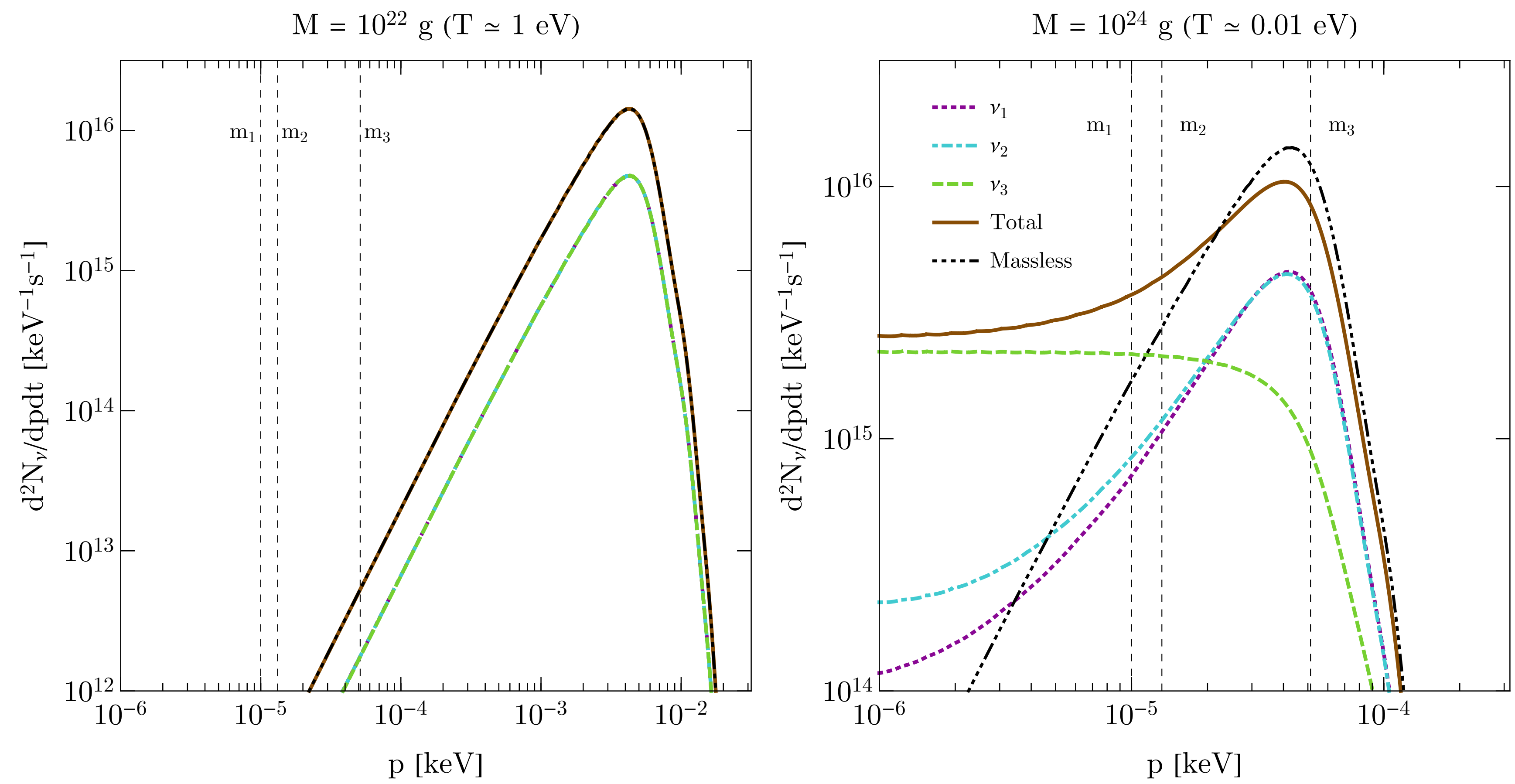

$$
\dot{M}=-\sum_{j} \frac{g_{j}}{2 \pi^{2}} \int d p E_{j}(p) \frac{\sigma_{\text {abs }}^{s_{j}}(M, p) p^{2}}{\exp \left[E_{j}(p) / T\right]-(-1)^{2 s_{j}}}
$$

